\title{
19. CHEMISTRY OF LEG 45 BASALTS
}

\author{
J. M. Rhodes, ${ }^{1}$ D. P. Blanchard, ${ }^{3}$ M. A. Dungan, ${ }^{4}$ K. V. Rodgers,${ }^{2}$ and J. C. Brannon ${ }^{2}$
}

\section{INTRODUCTION}

A total of 764 meters of volcanic basement was sampled during Leg 45 at Sites 395 and 396; most of the material recovered consists of basaltic pillow lavas, thin flows, and more massive cooling units. This paper is concerned with the major-and trace-element chemistry of these basalts. A companion paper by Dungan et al. (this volume) discusses the petrography, mineral chemistry, and 1-atmosphere melting experiments for the same suite of samples. The emphasis here will be on samples recovered from Holes 395 and 395A. Data for samples from Hole 396 are included for completeness, but will be reported in detail, together with data on samples from Hole 396B, in the Leg 46 Initial Reports.

Major- and trace-element analyses for 59 basalt samples from Holes 395 and $395 \mathrm{~A}$ are given in Table 1; analyses for 8 samples from Hole 396 are given in Table 2 . In these tables, the samples are listed in order of increasing depth within basement, reported to the nearest meter. Also indicated in Tables 1 and 2 are the major magmatic compositional units, first identified on the basis of shipboard X-ray fluorescence analyses, and modified slightly in the light of the recent data. Within these two tables, the oxidation ratio (O.R.) is given as $\mathrm{Fe}_{2} \mathrm{O}_{3} /($ total iron as $\mathrm{FeO}$ ). The atomic ratio $\mathrm{Mg}$ / $(\mathrm{Mg}+\mathrm{Fe})$ has been calculated, after adjustment for the oxidation state of iron, such that the ratio $\mathrm{Fe}^{3+}$ / (Total iron as $\mathrm{Fe}^{2+}$ ) is 0.1 (Bass, 1971). For convenience, the atomic $\mathrm{Mg} /(\mathrm{Mg}+\mathrm{Fe})$ ratio is designated the $\mathrm{Mg}^{\prime}$-value in Tables 1 and 2 and throughout the paper. The same convention for handling the oxidation state of iron has been used in the CIPW norm calculations that are referred to in the text and illustrated in Figure 1.

Table 3 presents additional trace-element data on 26 samples selected from Table 1 on the basis of petrography and major element chemistry.

The major-element data were obtained by X-ray fluorescence analysis (XRF) on fused glass discs, prepared by fusing the sample with a lanthanumbearing lithium borate fusion mixture (Norrish and Hutton, 1969). FeO was determined titrimetrically using the modified cold acid digestion method of Wilson (Maxwell, 1968), and $\mathrm{Fe}_{2} \mathrm{O}_{3}$ was obtained by difference from the XRF total iron value. $\mathrm{Na}_{2} \mathrm{O}$ was

\footnotetext{
'Lockheed Electronics Co., Inc., Houston, Texas. Present address: Dept. of Geol. and Geog., Univ. of Massachusetts, Amherst.

${ }^{2}$ Lockheed Electronics Co., Inc., Houston, Texas.

'NASA, Johnson Space Center, Houston, Texas.

'NASA, Research Associate, Johnson Space Center, Houston, Texas.
}

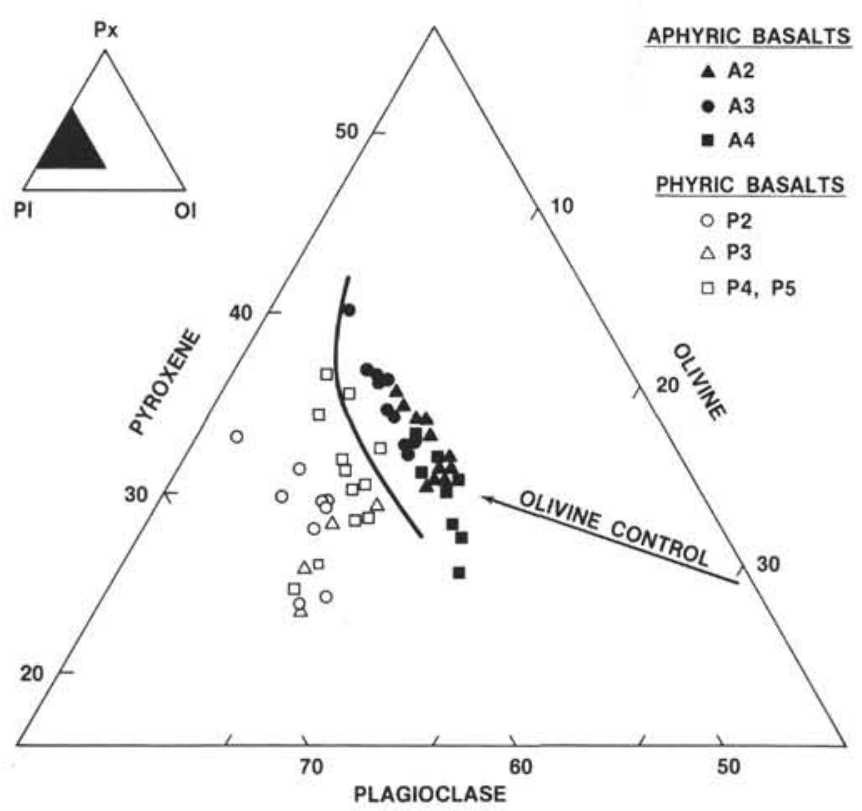

Figure 1. Normative olivine-plagioclase-pyroxene relationships in Leg 45 basalts. The classification and inferred olivine-plagioclase cotectic is from Shido et al. (1971).

determined on a separate 20 to $30 \mathrm{mg}$ aliquant by instrumental neutron-activation analysis (INAA). Total water content was measured coulometrically using a DuPont moisture analyzer, in which the sample is fused with a lead oxide flux.

The trace elements $(\mathrm{Rb}, \mathrm{Sr}, \mathrm{Y}, \mathrm{Zr}, \mathrm{Nb})$ were determined by XRF analysis on pressed powder pellets. Corrections were made for non-linear backgrounds, tube contamination, and inter-element interferences (Norrish and Chappell, 1967). Corrections for matrix effects were based on a modification of the Compton scattering method (Reynolds, 1967), using the AgCompton peak. Additional trace-element data (Table 3) ( $\mathrm{La}, \mathrm{Ce}, \mathrm{Sm}, \mathrm{Eu}, \mathrm{Tb}, \mathrm{Yb}, \mathrm{Lu}, \mathrm{Hf}, \mathrm{Cr}, \mathrm{Sc}, \mathrm{Ni})$ were obtained by INAA following the methods of Jacobs et al. (in press).

\section{BASALT CHEMISTRY}

All the basalts recovered from Holes 395, 395A, and 396 are olivine and hypersthene normative, containing between 1.8 and 14.2 per cent of normative olivine, and plotting in the olivine tholeiite field of the basalt tetrahedron (Yoder and Tilley, 1962). Alkali basalts and transitional tholeiites are conspicuously absent, as also are quartz tholeiites. They have the overall com- 
TABLE 1

Chemical Composition of Basalt From Site 395

\begin{tabular}{|c|c|c|c|c|c|}
\hline $\begin{array}{c}\text { Sample } \\
\text { (Interval in } \mathrm{cm} \text { ) }\end{array}$ & $395-11-1,42-44$ & $395-11-1,136-138$ & $395-11-2,100-105$ & $395-15-2,68-72$ & $395-16-2,63-66$ \\
\hline JSC No. & 109 & 110 & 111 & 112 & 113 \\
\hline Depth (m) & 93 & 94 & 95 & 133 & 142 \\
\hline Basalt type & $\mathrm{A}_{2} *$ & $\mathrm{~A}_{2} *$ & $\mathrm{~A}_{2}^{*}$ & $\mathrm{~A}_{2} *$ & $\mathrm{~A}_{2} *$ \\
\hline $\mathrm{SiO}_{2}$ & 49.4 & 49.3 & 48.5 & 49.3 & 49.6 \\
\hline $\mathrm{TiO}_{2}$ & 1.62 & 1.61 & 1.58 & 1.60 & 1.66 \\
\hline $\mathrm{Al}_{2} \mathrm{O}_{3}$ & 15.09 & 14.84 & 14.79 & 14.68 & 15.15 \\
\hline $\mathrm{Fe}_{2} \mathrm{O}_{3}$ & 1.28 & 1.36 & 1.81 & 2.61 & 4.62 \\
\hline $\mathrm{FeO}$ & 9.83 & 9.73 & 9.06 & 8.33 & 6.94 \\
\hline $\mathrm{MnO}$ & 0.20 & 0.19 & 0.17 & 0.18 & 0.19 \\
\hline $\mathrm{MgO}$ & 8.51 & 8.45 & 8.56 & 8.40 & 8.17 \\
\hline $\mathrm{CaO}$ & 10.41 & 10.34 & 10.18 & 10.17 & 10.43 \\
\hline $\mathrm{Na}_{2} \mathrm{O}$ & 2.80 & 2.92 & 2.82 & 2.89 & 2.82 \\
\hline $\mathrm{K}_{2} \mathrm{O}$ & 0.11 & 0.10 & 0.14 & 0.14 & 0.16 \\
\hline $\mathrm{P}_{2} \mathrm{O}_{5}$ & 0.13 & 0.11 & 0.12 & 0.13 & 0.16 \\
\hline $\mathrm{s}$ & 0.11 & 0.11 & 0.11 & 0.12 & 0.10 \\
\hline Total $\mathrm{H}_{2} \mathrm{O}$ & 0.55 & 0.43 & 1.56 & 1.42 & 0.91 \\
\hline Total & 100.04 & 99.50 & 99.37 & 99.94 & 100.94 \\
\hline Total $\mathrm{Fe}$ as $\mathrm{FeO}$ & 10.98 & 10.95 & 10.69 & 10.68 & 11.10 \\
\hline $\mathrm{Mg}^{\prime}$-value & 0.605 & 0.605 & 0.613 & 0.609 & 0.593 \\
\hline O. R. & 0.117 & 0.124 & 0.169 & 0.244 & 0.416 \\
\hline \multicolumn{6}{|l|}{$\begin{array}{l}\text { Trace Elements } \\
\text { (ppm) }\end{array}$} \\
\hline $\mathrm{Rb}$ & 1.2 & 0.9 & 1.7 & 1.1 & 1.1 \\
\hline $\mathrm{Sr}$ & 115 & 112 & 116 & 116 & 115 \\
\hline Y & 35 & 35 & 34 & 35 & 37 \\
\hline $\mathrm{Zr}_{\mathrm{r}}$ & 105 & 105 & 102 & 106 & 106 \\
\hline $\mathrm{Nb}$ & 2.4 & 1.9 & 1.9 & 1.7 & 1.8 \\
\hline $\begin{array}{c}\text { Sample } \\
\text { (Interval in cm) }\end{array}$ & $395 \mathrm{~A}-8-1,70-73$ & $395 \mathrm{~A}-9-1,73-79$ & $395 \mathrm{~A}-9-2,100-104$ & $395 \mathrm{~A}-10-1,144-146$ & $395 \mathrm{~A}-13-1,142-147$ \\
\hline JSC No. & 120 & 121 & 122 & 123 & 124 \\
\hline Depth (m) & 126 & 135 & 137 & 145 & 174 \\
\hline Basalt type & $\mathrm{A}_{2}$ & $\mathrm{~A}_{2}$ & $\mathrm{~A}_{2}$ & $\mathrm{~A}_{2}$ & $\mathrm{P}_{2}$ \\
\hline $\mathrm{SiO}_{2}$ & 48.7 & 48.5 & 48.9 & 48.8 & 49.2 \\
\hline $\mathrm{TiO}_{2}$ & 1.66 & 1.60 & 1.63 & 1.60 & 1.37 \\
\hline $\mathrm{Al}_{2} \mathrm{O}_{3}$ & 15.40 & 14.69 & 14.92 & 14.90 & 17.34 \\
\hline $\mathrm{Fe}_{2} \mathrm{O}_{3}$ & 2.56 & 2.84 & 2.67 & 1.97 & 2.52 \\
\hline $\mathrm{FeO}$ & 8.81 & 8.03 & 8.20 & 8.96 & 5.96 \\
\hline $\mathrm{MnO}$ & 0.20 & 0.20 & 0.19 & 0.19 & 0.15 \\
\hline $\mathrm{MgO}$ & 7.60 & 8.21 & 8.15 & 8.40 & 6.55 \\
\hline $\mathrm{CaO}$ & 10.46 & 10.17 & 10.29 & 10.23 & 11.54 \\
\hline $\mathrm{Na}_{2} \mathrm{O}$ & 2.90 & 2.93 & 3.01 & 2.90 & 2.83 \\
\hline $\mathrm{K}_{2} \mathrm{O}$ & 0.18 & 0.16 & 0.15 & 0.14 & 0.11 \\
\hline $\mathrm{P}_{2} \mathrm{O}_{5}$ & 0.17 & 0.12 & 0.13 & 0.12 & 0.10 \\
\hline $\mathrm{s}$ & 0.10 & 0.14 & 0.14 & 0.13 & 0.09 \\
\hline Total $\mathrm{H}_{2} \mathrm{O}$ & 1.62 & 1.93 & 1.25 & 1.41 & 1.94 \\
\hline Total & 100.33 & 99.56 & 99.61 & 99.79 & 99.66 \\
\hline Total $\mathrm{Fe}$ as $\mathrm{FeO}$ & 11.11 & 10.59 & 10.60 & 10.73 & 8.23 \\
\hline $\mathrm{Mg}^{\prime}$-value & 0.575 & 0.606 & 0.604 & 0.608 & 0.612 \\
\hline O. R. & 0.230 & 0.269 & 0.252 & 0.184 & 0.306 \\
\hline \multicolumn{6}{|l|}{$\begin{array}{l}\text { Trace Elements } \\
\text { (ppm) }\end{array}$} \\
\hline $\mathrm{Rb}$ & 1.6 & 1.5 & 1.3 & 1.3 & 1.1 \\
\hline $\mathrm{Sr}$ & 119 & 118 & 114 & 115 & 153 \\
\hline Y & 35 & 35 & 36 & 32 & 29 \\
\hline $\mathrm{Zr}$ & 104 & 103 & 106 & 103 & 94 \\
\hline $\mathrm{Nb}$ & 2.0 & 1.7 & 1.6 & 2.1 & 1.7 \\
\hline
\end{tabular}

positional characteristics typical of mid-ocean ridge tholeiites, as is indicated by their relatively constant $\mathrm{SiO}_{2}$ concentrations and low $\mathrm{TiO}_{2}$ abundances, together with low total alkali content and $\mathrm{K}_{2} \mathrm{O} / \mathrm{Na}_{2} \mathrm{O}$ .ratios (e.g., Engel et al., 1965; Melson et al., 1976).
$\mathrm{K}_{2} \mathrm{O}$ abundances are characteristically low in most of the samples $(<0.2 \%)$, and where comparative data are available, do not differ significantly from the $\mathrm{K}_{2} \mathrm{O}$ content of basaltic glass from the same samples. A few samples have higher $\mathrm{K}_{2} \mathrm{O}$ contents, not related to 
TABLE 1 - Continued

\begin{tabular}{|c|c|c|c|c|c|}
\hline $395-16-3,3-8$ & $395-18-2,43-46$ & $395-19-1,57-62$ & $395 \mathrm{~A}-41,66-69$ & $395 \mathrm{~A}-5-1,6-10$ & $395 \mathrm{~A}-6-1,130-134$ \\
\hline 114 & 115 & 116 & 117 & 118 & 119 \\
\hline 143 & 161 & 170 & 98 & 106 & 117 \\
\hline $\mathrm{A}_{2} *$ & $P_{1}^{*}$ & $\mathrm{P}_{2} *$ & $\mathrm{~A}_{3}$ & $\mathrm{~A}_{2}$ & $\mathrm{~A}_{2}$ \\
\hline 48.9 & 48.8 & 49.7 & 49.5 & 49.2 & 48.7 \\
\hline 1.60 & 1.14 & 1.29 & 1.70 & 1.63 & 1.60 \\
\hline 14.69 & 16.67 & 18.48 & 15.18 & 15.11 & 14.74 \\
\hline 2.20 & 2.90 & 3.23 & 2.86 & 3.95 & 4.30 \\
\hline 8.87 & 5.90 & 4.45 & 7.44 & 6.04 & 6.82 \\
\hline 0.18 & 0.15 & 0.12 & 0.17 & 0.17 & 0.18 \\
\hline 8.47 & 8.07 & 5.99 & 7.69 & 7.74 & 8.08 \\
\hline 10.25 & 12.24 & 12.10 & 11.17 & 11.03 & 10.11 \\
\hline 2.87 & 2.34 & 2.68 & 2.74 & 2.79 & 2.97 \\
\hline 0.17 & 0.13 & 0.11 & 0.28 & 0.26 & 0.22 \\
\hline 0.12 & 0.09 & 0.10 & 0.15 & 0.17 & 0.12 \\
\hline 0.14 & 0.05 & 0.03 & 0.07 & 0.05 & 0.07 \\
\hline 1.53 & 0.89 & 1.97 & 1.08 & 1.51 & 2.48 \\
\hline 99.94 & 99.38 & 100.27 & 100.04 & 99.64 & 100.45 \\
\hline 10.85 & 8.51 & 7.36 & 10.01 & 9.60 & 10.69 \\
\hline 0.607 & 0.653 & 0.617 & 0.603 & 0.615 & 0.599 \\
\hline 0.203 & 0.341 & 0.439 & 0.286 & 0.411 & 0.402 \\
\hline 1.1 & 1.5 & 1.2 & 2.6 & 3.3 & 3.0 \\
\hline 118 & 110 & 158 & 131 & 129 & 118 \\
\hline 36 & 26 & 26 & 36 & 35 & 34 \\
\hline 108 & 71 & 85 & 119 & 113 & 104 \\
\hline 2.1 & 1.1 & 2.0 & 2.3 & 1.9 & 2.0 \\
\hline
\end{tabular}

\begin{tabular}{|c|c|c|c|c|c|}
\hline $395 \mathrm{~A}-14-2,140-147$ & $395 \mathrm{~A}-14-3,29-34$ & $395 \mathrm{~A}-15-1,75-81$ & $395 \mathrm{~A}-15-4,10-15$ & $395 \mathrm{~A}-15-4,10-15$ & $395 \mathrm{~A}-15-4,99-107$ \\
\hline 125 & 126 & 127 & $128(1)$ & $128(2)$ & 129 \\
\hline 185 & 186 & 192 & 196 & 196 & 197 \\
\hline $\mathrm{P}_{2}$ & $\mathrm{P}_{2}$ & $\mathrm{P}_{2}$ & $\mathrm{P}_{2}$ & $\mathrm{P}_{2}$ & $\mathrm{P}_{2}$ \\
\hline 49.1 & 49.7 & 49.0 & 49.0 & 49.5 & 48.3 \\
\hline 1.34 & 1.33 & 1.35 & 1.36 & 1.35 & 1.30 \\
\hline 17.93 & 18.13 & 17.51 & 17.65 & 17.57 & 18.01 \\
\hline 1.57 & 2.40 & 2.69 & 1.40 & $(1.40)$ & 2.34 \\
\hline 6.94 & 5.86 & 5.97 & 7.25 & 7.25 & 5.96 \\
\hline 0.13 & 0.14 & 0.13 & 0.13 & 0.14 & 0.15 \\
\hline 6.81 & 6.58 & 6.97 & 7.07 & 7.03 & 6.82 \\
\hline 11.73 & 11.74 & 11.54 & 11.58 & 11.57 & 11.51 \\
\hline 2.80 & 2.85 & 2.76 & 2.71 & $(2.71)$ & 2.87 \\
\hline 0.09 & 0.07 & 0.11 & 0.11 & 0.11 & 0.08 \\
\hline 0.10 & 0.12 & 0.11 & 0.12 & 0.12 & 0.09 \\
\hline 0.10 & 0.12 & 0.12 & 0.11 & 0.11 & 0.08 \\
\hline 1.48 & 1.46 & 1.63 & 1.71 & - & 1.65 \\
\hline 100.12 & 100.49 & 99.87 & 100.21 & - & 99.20 \\
\hline 8.35 & 8.02 & 8.39 & 8.51 & 8.52 & 8.07 \\
\hline 0.618 & 0.619 & 0.622 & 0.622 & 0.620 & 0.626 \\
\hline 0.188 & 0.299 & 0.321 & 0.165 & - & 0.343 \\
\hline 0.9 & 0.5 & 1.0 & 1.3 & 1.0 & 0.5 \\
\hline 152 & 152 & 155 & 156 & 152 & 154 \\
\hline 27 & 27 & 30 & 29 & 29 & 28 \\
\hline 87 & 86 & 95 & 91 & 93 & 89 \\
\hline 1.2 & 1.9 & 1.4 & 1.4 & 1.8 & 1.7 \\
\hline
\end{tabular}

basalt type, which are presumably the result of seawater alteration, although this is not always correlated with total water content or the oxidation ratio.

The minor- and trace-element abundances are, similarly, within the range of typical mid-ocean ridge tholeiites (Kay et al., 1970; Schilling, 1971; Pearce and Cann, 1973; Hart, 1976; Bryan, et al., 1976; Erlank and Kable, 1976). This is illustrated by the chondritenormalized rare-earth patterns, which range between 13 and 24 times chondritic abundances for Sm, and which 
TABLE 1 - Continued

\begin{tabular}{|c|c|c|c|c|c|}
\hline $\begin{array}{c}\text { Sample } \\
\text { (Interval in cm) }\end{array}$ & $395 \mathrm{~A}-15-5,16-20$ & $395 \mathrm{~A}-17-1,46-55$ & $395 \mathrm{~A}-19-1,132-136$ & $395 \mathrm{~A}-21-1,87-90$ & $395 \mathrm{~A}-23-1,51-55$ \\
\hline JSC No. & 130 & 131 & 132 & 133 & 134 \\
\hline Depth (m) & 198 & 211 & 231 & 242 & 261 \\
\hline Basalt type & $\mathrm{P}_{2}$ & $\mathrm{P}_{3}$ & $\mathrm{P}_{3}$ & $\mathrm{P}_{3}$ & $\mathrm{P}_{4}$ \\
\hline $\mathrm{SiO}_{2}$ & 48.9 & 48.9 & 49.1 & 49.8 & 49.2 \\
\hline $\mathrm{TiO}_{2}$ & 1.14 & 1.09 & 1.08 & 1.03 & 1.12 \\
\hline $\mathrm{Al}_{2} \mathrm{O}_{3}$ & 19.16 & 18.05 & 16.85 & 17.73 & 18.51 \\
\hline $\mathrm{Fe}_{2} \mathrm{O}_{3}$ & 1.47 & 3.70 & 3.42 & 2.58 & 3.76 \\
\hline $\mathrm{FeO}$ & 6.19 & 4.78 & 4.80 & 5.23 & 4.24 \\
\hline $\mathrm{MnO}$ & 0.13 & 0.15 & 0.13 & 0.14 & 0.14 \\
\hline $\mathrm{MgO}$ & 6.96 & 6.66 & 7.61 & 7.53 & 6.51 \\
\hline $\mathrm{CaO}$ & 11.83 & 12.83 & 12.19 & 12.49 & 12.59 \\
\hline $\mathrm{Na}_{2} \mathrm{O}$ & 2.75 & 2.50 & 2.31 & 2.39 & 2.75 \\
\hline $\mathrm{K}_{2} \mathrm{O}$ & 0.07 & 0.12 & 0.13 & 0.15 & 0.15 \\
\hline $\mathrm{P}_{2} \mathrm{O}_{5}$ & 0.09 & 0.09 & 0.08 & 0.10 & 0.12 \\
\hline$S^{2}$ & 0.09 & 0.01 & 0.01 & 0.03 & 0.01 \\
\hline Total $\mathrm{H}_{2} \mathrm{O}$ & 1.63 & 1.19 & 1.94 & 1.68 & 1.01 \\
\hline Total & 100.41 & 100.08 & 99.67 & 100.91 & 100.10 \\
\hline Total $\mathrm{Fe}$ as $\mathrm{FeO}$ & 7.51 & 8.11 & 7.88 & 7.55 & 7.62 \\
\hline Mg'-value & 0.647 & 0.619 & 0.657 & 0.664 & 0.629 \\
\hline O.R. & 0.196 & 0.456 & 0.434 & 0.342 & 0.493 \\
\hline \multicolumn{6}{|l|}{$\begin{array}{l}\text { Trace Elements } \\
\text { (ppm) }\end{array}$} \\
\hline $\mathrm{Rb}$ & 0.6 & 1.9 & 2.1 & 1.7 & 1.4 \\
\hline $\mathrm{Sr}$ & 153 & 119 & 110 & 112 & 138 \\
\hline $\mathrm{Y}$ & 24 & 24 & 23 & 22 & 24 \\
\hline $\mathrm{Zr}_{\mathrm{r}}$ & 76 & 65 & 64 & 60 & 68 \\
\hline $\mathrm{Nb}$ & 0.7 & 0.9 & 1.2 & 1.5 & 1.3 \\
\hline $\begin{array}{c}\text { Sample } \\
\text { (Interval in cm) }\end{array}$ & $395 A-31-1,96-107$ & $395 \mathrm{~A}-33-1,142-148$ & $395 \mathrm{~A}-34-1,140-143$ & $395 \mathrm{~A}-35-1,26-32$ & $395 \mathrm{~A}-36-1,46-50$ \\
\hline JSC No. & 142 & 143 & 144 & 145 & 146 \\
\hline Depth (m) & 337 & 355 & 364 & 373 & 383 \\
\hline Basalt type & $\mathrm{P}_{5}$ & $\mathrm{P}_{5}$ & $\mathrm{~A}_{3}$ & $\mathrm{~A}_{3}$ & $\mathrm{~A}_{3}$ \\
\hline $\mathrm{SiO}_{2}$ & 49.8 & 48.9 & 49.5 & 49.3 & 48.9 \\
\hline $\mathrm{TiO}_{2}$ & 1.10 & 1.14 & 1.69 & 1.67 & 1.67 \\
\hline $\mathrm{Al}_{2} \mathrm{O}_{3}$ & 18.62 & 17.55 & 15.32 & 1.501 & 15.13 \\
\hline $\mathrm{Fe}_{2} \mathrm{O}_{3}$ & 3.28 & 4.08 & 2.98 & 4.50 & 4.10 \\
\hline $\mathrm{FeO}$ & 4.49 & 4.00 & 7.24 & 5.66 & 5.80 \\
\hline $\mathrm{MnO}$ & 0.12 & 0.14 & 0.19 & 0.16 & 0.19 \\
\hline $\mathrm{MgO}$ & 6.69 & 7.11 & 7.61 & 7.45 & 7.48 \\
\hline $\mathrm{CaO}$ & 12.17 & 11.85 & 11.21 & 10.95 & 11.09 \\
\hline $\mathrm{Na}_{2} \mathrm{O}$ & 2.89 & 2.73 & 2.80 & 2.88 & 2.93 \\
\hline $\mathrm{K}_{2} \mathrm{O}$ & 0.18 & 0.18 & 0.22 & 0.32 & 0.26 \\
\hline $\mathrm{P}_{2} \mathrm{O}_{5}$ & 0.08 & 0.11 & 0.17 & 0.13 & 0.14 \\
\hline $\mathrm{S}^{-}$ & 0.01 & 0.01 & 0.09 & 0.03 & 0.02 \\
\hline Total $\mathrm{H}_{2} \mathrm{O}$ & 1.77 & 1.86 & 1.33 & 1.95 & 2.28 \\
\hline Total & 101.23 & 99.66 & 100.32 & 100.03 & 100.03 \\
\hline Total $\mathrm{Fe}$ as $\mathrm{FeO}$ & 7.44 & 7.67 & 9.92 & 9.71 & 9.49 \\
\hline $\mathrm{Mg}^{\prime}$-value & 0.614 & 0.647 & 0.603 & 0.603 & 0.609 \\
\hline O. R. & 0.440 & 0.532 & 0.300 & 0.463 & 0.432 \\
\hline \multicolumn{6}{|l|}{$\begin{array}{l}\text { Trace Elements } \\
\text { (ppm) }\end{array}$} \\
\hline $\mathrm{Rb}$ & 2.6 & 2.5 & 1.0 & 4.7 & 3.3 \\
\hline $\mathrm{Sr}$ & 175 & 151 & 127 & 133 & 130 \\
\hline $\mathrm{Y}$ & 23 & 23 & 36 & 35 & 36 \\
\hline $\mathrm{Zr}$ & 71 & 73 & 116 & 117 & 117 \\
\hline $\mathrm{Nb}$ & 0.8 & 1.0 & 2.4 & 2.0 & 1.9 \\
\hline
\end{tabular}

display the light rare-earth depletion typical of "normal" or type I ocean ridge basalts (Bryan, et al., 1976). $\mathrm{Mg}^{\prime}$-values in these rocks vary from about 0.57 to 0.67 (Figure 2), which is within the range prevalent for midocean ridge basalts but is lower than the values found in the most primitive basalts so far identified (Frey et al.,
1974; Bryan and Moore, 1976; Rhodes et al., in press). These values, together with low $\mathrm{Ni}$ concentrations, moderately high magmaphile element abundances, and the presence of multiple phenocryst phases (Dungan et al., this volume), are taken as evidence that these basalts have all undergone a substantial differentiation history. 
TABLE 1 - Continued

\begin{tabular}{|c|c|c|c|c|c|}
\hline $395 \mathrm{~A}-24-2,69-72$ & $395 \mathrm{~A}-25-1,96-100$ & $395 \mathrm{~A}-26-2,125-129$ & $395 \mathrm{~A}-27-2,111-116$ & $395 \mathrm{~A}-38-1,105-112$ & $395 \mathrm{~A}-29-1,125-131$ \\
\hline 135 & 136 & 137 & 138 & 139 & 141 \\
\hline 272 & 280 & 292 & 301 & 309 & 317 \\
\hline $\mathrm{P}_{4}$ & $\mathrm{P}_{4}$ & $\mathrm{P}_{4}$ & $\mathrm{P}_{4}$ & $\mathrm{P}_{5}$ & $\mathrm{P}_{5}$ \\
\hline 49.1 & 49.3 & 38.9 & 49.6 & 48.8 & 49.8 \\
\hline 1.15 & 1.14 & 1.25 & 1.14 & 1.19 & 1.14 \\
\hline 17.26 & 17.10 & 18.85 & 17.13 & 19.09 & 17.07 \\
\hline 2.80 & 2.58 & 3.41 & 1.94 & .307 & 2.65 \\
\hline 5.11 & 5.28 & 4.85 & 6.03 & 4.97 & 5.73 \\
\hline 0.15 & 0.14 & 0.17 & 0.15 & 0.15 & 0.15 \\
\hline 7.90 & 7.67 & 5.87 & 8.03 & 6.33 & 8.00 \\
\hline 12.17 & 11.99 & 12.27 & 12.08 & 12.22 & 12.04 \\
\hline 2.65 & 2.62 & 2.84 & 2.64 & 2.78 & 2.59 \\
\hline 0.13 & 0.14 & 0.16 & 0.15 & 0.15 & 0.13 \\
\hline 0.09 & 0.11 & 0.13 & 0.10 & 0.11 & 0.10 \\
\hline 0.00 & 0.01 & 0.02 & 0.02 & 0.02 & 0.02 \\
\hline 0.88 & 1.54 & 1.26 & 0.71 & 0.97 & 0.83 \\
\hline 99.42 & 99.58 & 99.96 & 99.69 & 99.87 & 99.41 \\
\hline 7.63 & 7.60 & 7.92 & 7.78 & 7.73 & 8.11 \\
\hline 0.672 & 0.667 & 0.595 & 0.671 & 0.619 & 0.661 \\
\hline 0.367 & 0.339 & 0.431 & 0.249 & 0.397 & 0.327 \\
\hline 1.5 & 2.3 & 2.5 & 2.1 & 2.7 & 2.0 \\
\hline 131 & 133 & 165 & 129 & 159 & 132 \\
\hline 25 & 24 & 26 & 25 & 27 & 25 \\
\hline 72 & 73 & 82 & 73 & 82 & 74 \\
\hline 1.5 & 0.9 & 1.3 & 1.0 & 1.1 & 1.3 \\
\hline
\end{tabular}

\begin{tabular}{|c|c|c|c|c|c|}
\hline $395 \mathrm{~A}-38-1,142-148$ & $395 \mathrm{~A}-41-1,107-111$ & $395 \mathrm{~A}-46-1,61-64$ & $395 \mathrm{~A}-49-1,26-31$ & $395 \mathrm{~A}-50-2,143-147$ & $395 \mathrm{~A}-51-3,78-82$ \\
\hline 147 & 148 & 149 & 150 & 151 & 152 \\
\hline 393 & 428 & 476 & 504 & 516 & 526 \\
\hline $\mathrm{A}_{3}$ & $\mathrm{~A}_{3}$ & $\mathrm{~A}_{3}$ & $\mathrm{~A}_{3}$ & $\mathrm{~A}_{3}$ & $\mathrm{~A}_{4}$ \\
\hline 48.6 & 49.7 & 49.3 & 49.5 & 48.8 & 48.8 \\
\hline 1.65 & 1.70 & 1.75 & 1.74 & 1.72 & 1.70 \\
\hline 14.61 & 15.30 & 15.10 & 15.13 & 15.07 & 14.93 \\
\hline 4.15 & 2.90 & 3.90 & 3.14 & 3.97 & 3.52 \\
\hline 5.83 & 7.28 & 6.29 & 7.24 & 6.38 & 6.67 \\
\hline 0.17 & 0.17 & 0.19 & 0.19 & 0.16 & 0.18 \\
\hline 7.40 & 7.28 & 7.21 & 7.29 & 7.67 & 7.69 \\
\hline 10.83 & 11.14 & 11.10 & 11.19 & 11.02 & 10.87 \\
\hline 2.89 & 2.82 & 2.90 & 2.84 & 2.85 & 2.88 \\
\hline 0.22 & 0.22 & 0.31 & 0.18 & 0.22 & 0.26 \\
\hline 0.14 & 0.15 & 0.17 & 0.17 & 0.16 & 0.15 \\
\hline 0.004 & 0.08 & 0.02 & 0.09 & 0.05 & 0.07 \\
\hline 2.00 & 1.11 & 1.26 & 1.30 & 1.76 & 1.77 \\
\hline 98.53 & 99.85 & 99.50 & 99.97 & 99.84 & 99.51 \\
\hline 9.56 & 9.89 & 9.80 & 10.07 & 9.95 & 9.84 \\
\hline 0.605 & 0.593 & 0.593 & 0.589 & 0.604 & 0.607 \\
\hline 0.434 & 0.293 & 0.227 & 0.312 & 0.399 & 0.358 \\
\hline 2.3 & 0.7 & 4.5 & 1.5 & 2.8 & 2.3 \\
\hline 134 & 129 & 132 & 131 & $130^{\circ}$ & 129 \\
\hline 35 & 36 & 37 & 37 & 37 & 37 \\
\hline 114 & 117 & 125 & 121 & 121 & 122 \\
\hline 2.0 & 2.1 & 2.2 & 2.5 & 1.9 & 2.1 \\
\hline
\end{tabular}

Most samples selected for analysis were relatively fresh, with total water contents usually less than 2 per cent and oxidation ratios mostly below 0.4 . It is unlikely, therefore, that the major- or trace-element chemistry of these samples will have been significantly modified by seawater alteration. The mobile elements
$\mathrm{K}, \mathrm{Rb}$, and $\mathrm{S}$ are notable exceptions. The effects of alteration on sulfur are especially pronounced, and there is a marked inverse correlation between the oxidation ratio and the sulfur content (Figure 3 ). It would appear from this relationship that unaltered samples should contain about 0.14 per cent sulfur, a 
TABLE 1 - Continued

\begin{tabular}{|c|c|c|c|c|c|}
\hline $\begin{array}{c}\text { Sample } \\
\text { (Interval in cm) }\end{array}$ & $395 \mathrm{~A}-52-2,75-80$ & $395 \mathrm{~A}-56-3,62-65$ & $395 \mathrm{~A}-57-1,40-43$ & $395 \mathrm{~A}-59-1,67-70$ & $395 \mathrm{~A}-60-1,66-70$ \\
\hline JSC No. & 153 & 154 & 155 & 156 & 157 \\
\hline Depth (m) & 534 & 565 & 570 & 590 & 599 \\
\hline Basalt type & $\mathrm{A}_{3}$ & $\mathrm{~A}_{3}$ & $\mathrm{~A}_{4}$ & $\mathrm{~A} 4$ & $\mathrm{~A}_{4}$ \\
\hline $\mathrm{SiO}_{2}$ & 50.3 & 49.4 & 48.8 & 48.3 & 48.4 \\
\hline $\mathrm{TiO}_{2}$ & 1.73 & 1.75 & 1.57 & 1.58 & 1.59 \\
\hline $\mathrm{Al}_{2} \mathrm{O}_{3}$ & 15.22 & 14.99 & 14.93 & 14.93 & 15.12 \\
\hline $\mathrm{Fe}_{2} \mathrm{O}_{3}$ & 2.94 & 3.21 & 2.32 & 2.69 & 3.21 \\
\hline $\mathrm{FeO}$ & 7.37 & 7.12 & 8.02 & 7.65 & 6.94 \\
\hline $\mathrm{MnO}$ & 0.19 & 0.18 & 0.19 & 0.16 & 0.19 \\
\hline $\mathrm{MgO}$ & 7.34 & 7.82 & 8.62 & 8.45 & 8.01 \\
\hline $\mathrm{CaO}$ & 11.10 & 10.82 & 10.55 & 10.71 & 10.77 \\
\hline $\mathrm{Na}_{2} \mathrm{O}$ & 2.76 & 2.80 & 2.80 & 2.83 & 2.85 \\
\hline $\mathrm{K}_{2} \mathrm{O}$ & 0.19 & 0.19 & 0.15 & 0.13 & 0.14 \\
\hline $\mathrm{P}_{2} \mathrm{O}_{5}$ & 0.16 & 0.18 & 0.15 & 0.13 & 0.12 \\
\hline $\mathrm{s}$ & 0.11 & 0.07 & 0.09 & 0.05 & 0.07 \\
\hline Total $\mathrm{H}_{2} \mathrm{O}$ & 1.38 & 1.44 & 1.50 & 1.84 & 1.95 \\
\hline Total & 100.75 & 100.02 & 99.51 & 99.42 & 99.36 \\
\hline Total $\mathrm{Fe}$ as $\mathrm{FeO}$ & 10.02 & 10.01 & 10.11 & 10.07 & 9.83 \\
\hline $\mathrm{Mg}^{\prime}$-value & 0.592 & 0.607 & 0.628 & 0.624 & 0.617 \\
\hline O. R. & 0.293 & 0.321 & 0.229 & 0.267 & 0.326 \\
\hline \multicolumn{6}{|l|}{$\begin{array}{c}\text { Trace Elements } \\
(\mathrm{ppm})\end{array}$} \\
\hline $\mathrm{Rb}$ & 1.0 & 1.2 & 0.9 & 0.6 & 0.9 \\
\hline $\mathrm{Sr}$ & 128 & 127 & 121 & 125 & 125 \\
\hline Y & 37 & 38 & 34 & 34 & 35 \\
\hline $\mathrm{Zr}$ & 119 & 125 & 107 & 109 & 109 \\
\hline $\mathrm{Nb}$ & 2.8 & 2.7 & 2.4 & 2.4 & 1.8 \\
\hline
\end{tabular}

Sample

\begin{tabular}{lccccc} 
(Interval in cm) & $395 \mathrm{~A}-64-4,130-135$ & $395 \mathrm{~A}-64, \mathrm{CC}, 17-21$ & $395 \mathrm{~A}-66-1,36-41$ & $395 \mathrm{~A}-66-3,12-16$ & $395 \mathrm{~A}-67, \mathrm{CC}, 130-135$ \\
\hline JSC No. & 164 & 165 & 166 & 167 & 168
\end{tabular}

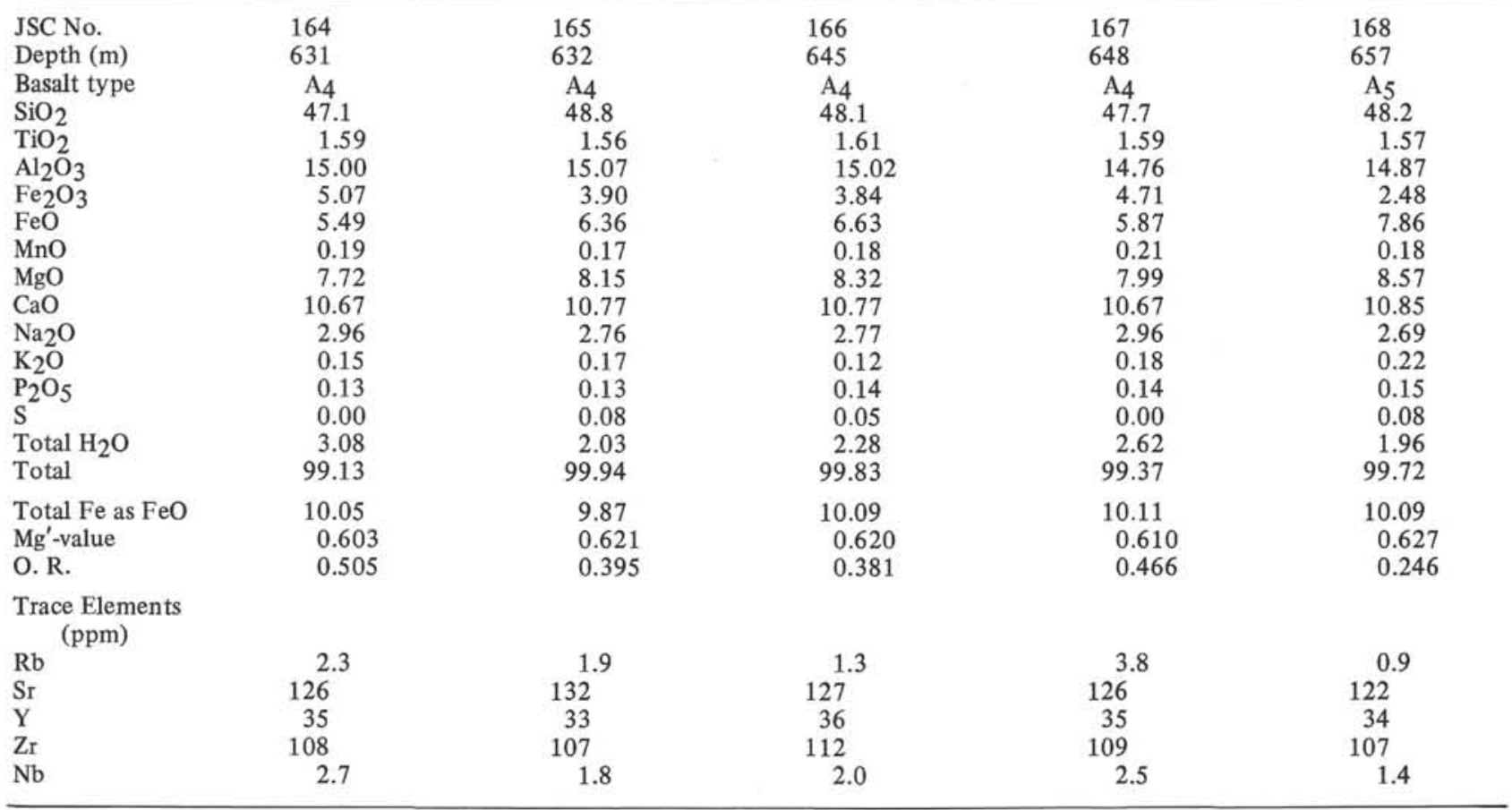

value somewhat larger than that proposed by Moore and Fabbi (1971) as typical for unaltered basalts recovered from deep water.

Two broad basalt types, differing fundamentally in both petrography and whole-rock chemistry, prevail at Site 395 . These are, respectively, the aphyric and phyric basalts. The aphyric basalts lack megascopic phenocrysts, but contain microphenocrysts of olivine and plagioclase; the phyric basalts contain abundant (10 to $30 \%$ ) plagioclase phenocrysts, together with phenocrysts of olivine, rarer $\mathrm{Cr}$-diopside, and minor chromian spinel. The aphyric basalts are the more 
TABLE 1 - Continued

\begin{tabular}{|c|c|c|c|c|c|}
\hline $395 \mathrm{~A}-60-3,33-37$ & $395 \mathrm{~A}-61-1,142-150$ & $395 \mathrm{~A}-62-1,10-15$ & $395 \mathrm{~A}-63-1,42-47$ & $395 \mathrm{~A}-63-4,86-93$ & $395 \mathrm{~A}-64-1,29-37$ \\
\hline 158 & 159 & 160 & 161 & 162 & 163 \\
\hline 602 & 608 & 617 & 619 & 624 & 626 \\
\hline $\mathrm{A}_{4}$ & $\mathrm{P}_{4}^{\prime}$ & $\mathrm{P}_{4}^{\prime}$ & $\mathrm{P}_{4}^{\prime}$ & $\mathrm{P}_{4}^{\prime}$ & $\mathrm{P}_{4}^{\prime}$ \\
\hline 47.2 & 49.2 & 49.3 & 49.6 & 48.5 & 49.1 \\
\hline 1.55 & 1.21 & 1.09 & 1.12 & 1.06 & 1.06 \\
\hline 15.09 & 16.05 & 17.15 & 16.47 & 17.19 & 17.21 \\
\hline 5.02 & 3.81 & 2.10 & 2.68 & 3.92 & 3.27 \\
\hline 5.36 & 4.92 & 5.93 & 5.56 & 4.16 & 4.71 \\
\hline 0.18 & 0.15 & 0.16 & 0.17 & 0.15 & 0.16 \\
\hline 8.40 & 7.84 & 7.93 & 8.18 & 7.56 & 7.31 \\
\hline 10.60 & 11.71 & 11.63 & 11.59 & 11.77 & 12.45 \\
\hline 2.77 & 2.72 & 2.70 & 2.54 & 2.64 & 4.21 \\
\hline 0.16 & 0.16 & 0.08 & 0.09 & 0.12 & 0.10 \\
\hline 0.13 & 0.10 & 0.08 & 0.09 & 0.09 & 0.08 \\
\hline 0.01 & 0.01 & 0.11 & 0.05 & 0.00 & 0.02 \\
\hline 2.17 & 1.66 & 1.75 & 1.78 & 2.75 & 1.31 \\
\hline 98.61 & 99.55 & 100.02 & 99.92 & 99.90 & 100.87 \\
\hline 9.88 & 8.35 & 7.82 & 7.97 & 7.69 & 7.65 \\
\hline 0.627 & 0.650 & 0.668 & 0.670 & 0.661 & 0.654 \\
\hline 0.508 & 0.456 & 0.269 & 0.336 & 0.510 & 0.427 \\
\hline 2.2 & 3.9 & 0.8 & 1.0 & 4.0 & 1.7 \\
\hline 117 & 128 & 122 & 123 & 130 & 136 \\
\hline 34 & 27 & 23 & 24 & 22 & 23 \\
\hline 103 & 78 & 69 & 71 & 65 & 68 \\
\hline 2.3 & 0.6 & 1.1 & 0.3 & 0.8 & 0.9 \\
\hline
\end{tabular}

TABLE 2

Chemical Composition of Basalt From Site 396

\begin{tabular}{|c|c|c|c|c|c|c|c|}
\hline $\begin{array}{c}\text { Sample } \\
\text { (Interval in cm) }\end{array}$ & $15-1,110-115$ & $16-1,97-101$ & $18-3,147-150$ & $22-2,120-128$ & $23-1,58-61$ & $24-2,49-54$ & $25-1,134-139$ \\
\hline JSC No. & 173 & 176 & 175 & 171 & 172 & 169 & 170 \\
\hline Depth (m) & 127 & 137 & 159 & 193 & 201 & 211 & 221 \\
\hline $\mathrm{SiO}_{2}$ & 49.30 & 48.79 & 49.27 & 48.99 & 49.17 & 49.81 & 49.30 \\
\hline $\mathrm{TiO}_{2}$ & 1.27 & 1.26 & 1.29 & 1.23 & 1.25 & 1.30 & 1.29 \\
\hline $\mathrm{Al}_{2} \mathrm{O}_{3}$ & 16.95 & 16.86 & 16.30 & 16.69 & 17.29 & 16.52 & 16.21 \\
\hline $\mathrm{Fe}_{2} \mathrm{O}_{3}$ & 1.39 & 3.64 & 3.85 & 3.65 & 4.33 & 3.92 & 4.43 \\
\hline $\mathrm{FeO}$ & 7.15 & 4.90 & 4.72 & 4.68 & 4.19 & 5.02 & 4.68 \\
\hline $\mathrm{MnO}$ & 0.17 & 0.15 & 0.14 & 0.14 & 0.13 & 0.17 & 0.17 \\
\hline $\mathrm{MgO}$ & 7.48 & 8.03 & 7.66 & 7.60 & 6.42 & 7.21 & 6.86 \\
\hline $\mathrm{CaO}$ & 11.80 & 11.85 & 11.48 & 11.66 & 12.07 & 11.76 & 11.69 \\
\hline $\mathrm{Na}_{2} \mathrm{O}$ & 2.66 & 2.87 & 2.75 & 2.79 & 2.77 & 2.72 & 2.68 \\
\hline $\mathrm{K}_{2} \mathrm{O}$ & 0.20 & 0.21 & 0.23 & 0.24 & 0.20 & 0.23 & 0.25 \\
\hline $\mathrm{P}_{2} \mathrm{O}_{5}$ & 0.09 & 0.08 & 0.08 & 0.09 & 0.09 & 0.09 & 0.15 \\
\hline $\mathrm{s}^{2}$ & 0.05 & 0.05 & 0.02 & 0.02 & 0.02 & 0.01 & 0.03 \\
\hline Total $\mathrm{H}_{2} \mathrm{O}$ & 1.49 & 1.52 & 2.28 & 2.47 & 2.86 & 2.10 & 2.52 \\
\hline Total & 100.00 & 100.21 & 100.70 & 100.24 & 100.79 & 100.86 & 100.26 \\
\hline Total $\mathrm{Fe}$ as $\mathrm{Fe} 0$ & 8.40 & 8.17 & 8.18 & 7.96 & 8.09 & 8.55 & 8.68 \\
\hline $\mathrm{Mg}^{\prime}$-value & 0.638 & 0.661 & 0.650 & 0.654 & 0.611 & 0.626 & 0.610 \\
\hline O.R. & 0.165 & 0.445 & 0.471 & 0.459 & 0.535 & 0.459 & 0.510 \\
\hline \multicolumn{8}{|l|}{$\begin{array}{c}\text { Trace Elements } \\
\text { (ppm) }\end{array}$} \\
\hline $\mathrm{Rb}$ & 1.0 & 2.5 & 2.7 & 3.3 & 2.9 & 3.5 & \\
\hline $\mathrm{Sr}$ & 149 & 145 & 160 & 145 & 152 & 127 & \\
\hline $\mathrm{Y}$ & 26 & 27 & 28 & 26 & 27 & 28 & \\
\hline $\mathrm{Z}_{\mathrm{r}}$ & 84 & 85 & 87 & 83 & 79 & 83 & \\
\hline $\mathrm{Nb}$ & 2.1 & 1.4 & 1.6 & 2.0 & 1.1 & 1.4 & \\
\hline
\end{tabular}

abundant, and comprise about 59 per cent of the cored interval.

Chemically, the phyric basalts are distinguished from the aphyric basalts by higher $\mathrm{Al}_{2} \mathrm{O}_{3}$ (16.5 to $19.2 \%)$ and $\mathrm{CaO}(11.5$ to $12.8 \%)$ concentrations, and by lower $\mathrm{SiO}_{2}, \mathrm{FeO}$, and $\mathrm{MgO}$ concentrations (Figures 4 and 5). These differences are reflected in higher normative plagioclase contents and in lower $\mathrm{Ab} /$ $(\mathrm{Ab}+\mathrm{An})$ ratios in the phyric basalts. Although both total iron and $\mathrm{MgO}$ are lower in the phyric basalts, 
TABLE 3

Trace Element Abundances (ppm) in Selected Leg 45 Samples

\begin{tabular}{|c|c|c|c|c|c|c|}
\hline $\begin{array}{c}\text { Sample } \\
\text { (Interval in } \mathrm{cm} \text { ) }\end{array}$ & $395-11-1,42-44$ & $395-11-2,100-105$ & $395-19-1,57-62$ & $395 \mathrm{~A}-4-1,66-69$ & $395 \mathrm{~A}-8-1,70-73$ & $395 \mathrm{~A}-10-1,144-146$ \\
\hline JSC No. & 109 & 111 & 116 & 117 & 120 & 123 \\
\hline Depth (m) & 95 & 101 & 173 & 99 & 129 & 153 \\
\hline Basalt type & $\mathrm{A}_{2}$ & $\mathrm{~A}_{2}$ & $\mathrm{P}_{2}$ & $\mathrm{~A}_{3}$ & $\mathrm{~A}_{2}$ & $\mathrm{~A}_{2}$ \\
\hline $\mathrm{Sc}$ & 36.6 & 36.0 & 29.8 & 38.4 & 37.9 & 37.1 \\
\hline $\mathrm{C}_{\mathrm{I}}$ & 290 & 290 & 252 & 320 & 300 & 300 \\
\hline $\mathrm{Ni}$ & 120 & 190 & 110 & 140 & 140 & 150 \\
\hline $\mathrm{La}$ & 2.91 & 2.79 & 2.71 & 3.77 & 3.18 & 3.31 \\
\hline $\mathrm{Ce}$ & 9.6 & 9.7 & 9.3 & 12.2 & 11.1 & 9.8 \\
\hline $\mathrm{Sm}$ & 3.38 & 3.41 & 3.09 & 3.91 & 3.72 & 3.68 \\
\hline Eu & 1.33 & 1.27 & 1.06 & 1.40 & 1.39 & 1.34 \\
\hline $\mathrm{Tb}$ & 0.92 & 0.92 & 0.76 & 1.0 & 0.95 & 0.95 \\
\hline $\mathrm{Yb}$ & 3.3 & 3.2 & 2.8 & 3.5 & 3.6 & 3.7 \\
\hline $\mathrm{Lu}$ & 0.50 & 0.47 & 0.42 & 0.53 & 0.52 & 0.56 \\
\hline $\mathrm{Hf}$ & 3.0 & 2.7 & 2.3 & 3.0 & 3.0 & 2.9 \\
\hline
\end{tabular}

\begin{tabular}{|c|c|c|c|c|c|c|}
\hline $\begin{array}{c}\text { Sample } \\
\text { (Interval in cm) }\end{array}$ & $395 \mathrm{~A}-25-1,96-100$ & $395 \mathrm{~A}-27-2,111-116$ & $395 \mathrm{~A}-31-1,96-107$ & $395 \mathrm{~A}-34-1,140-143$ & $395 \mathrm{~A}-41-1,107-111$ & $395 \mathrm{~A}-49-1,26-31$ \\
\hline JSC No. & 136 & 138 & 142 & 144 & 148 & 150 \\
\hline Depth (m) & 285 & 306 & 339 & 372 & 433 & 504 \\
\hline Basalt type & $\mathrm{P}_{4}$ & $\mathrm{P}_{4}$ & $\mathrm{P}_{5}$ & $\mathrm{~A}_{3}$ & $\mathrm{~A}_{3}$ & $\mathrm{~A}_{3}$ \\
\hline $\mathrm{Sc}$ & 33.8 & 33.5 & 32.3 & 38.5 & 38.7 & 38.8 \\
\hline $\mathrm{Cr}_{\mathrm{r}}$ & 340 & 340 & 310 & 280 & 290 & 290 \\
\hline $\mathrm{Ni}$ & 150 & 130 & 140 & 110 & 100 & 40 \\
\hline $\mathrm{La}$ & 2.41 & 2.33 & 2.48 & 3.82 & 3.70 & 4.14 \\
\hline $\mathrm{Ce}$ & 8.1 & 7.2 & 8.1 & 11.7 & 12.3 & 12.5 \\
\hline $\mathrm{Sm}$ & 2.77 & 2.72 & 2.82 & 4.10 & 4.09 & 4.28 \\
\hline Eu & 1.06 & 1.04 & 1.06 & 1.43 & 1.47 & 1.45 \\
\hline $\mathrm{Tb}$ & 0.72 & 0.60 & 0.67 & 0.99 & 1.05 & 1.09 \\
\hline $\mathrm{Yb}$ & 2.5 & 2.7 & 2.7 & 3.7 & 3.6 & 3.8 \\
\hline $\mathrm{Lu}$ & 0.40 & 0.40 & 0.41 & 0.56 & 0.55 & 0.56 \\
\hline $\mathrm{Hf}$ & 2.3 & 2.2 & 2.2 & 3.3 & 3.3 & 3.2 \\
\hline
\end{tabular}

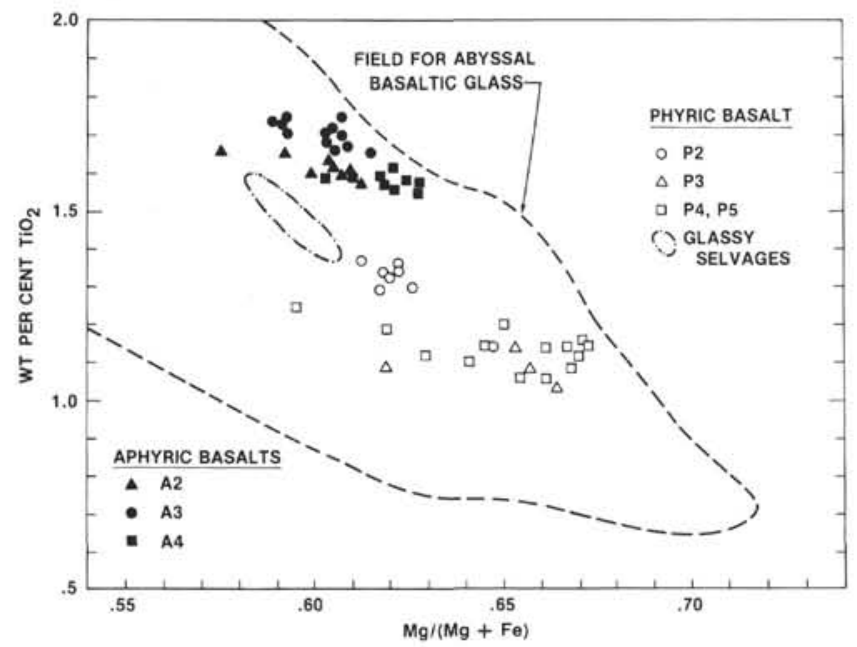

Figure 2. $\mathrm{TiO}_{2}$ versus $\mathrm{Mg}^{\prime}$-value for Leg 45 basalts.

The field for abyssal volcanic glass compositions is taken from the data of Melson et al. (1976).

$\mathrm{Mg}^{\prime}$-values tend to be higher, an indication that they are more "primitive" than the aphyric basalts (Figures 2 and 5). $\mathrm{TiO}_{2}$, and most other minor- and magmaphile trace-element abundances are markedly lower in the phyric basalts (Figure 2). This difference extends also to the glassy rims of the phyric basalts, and is therefore not simply a consequence of plagioclase dilution. Strontium, on the other hand, is higher in the phyric

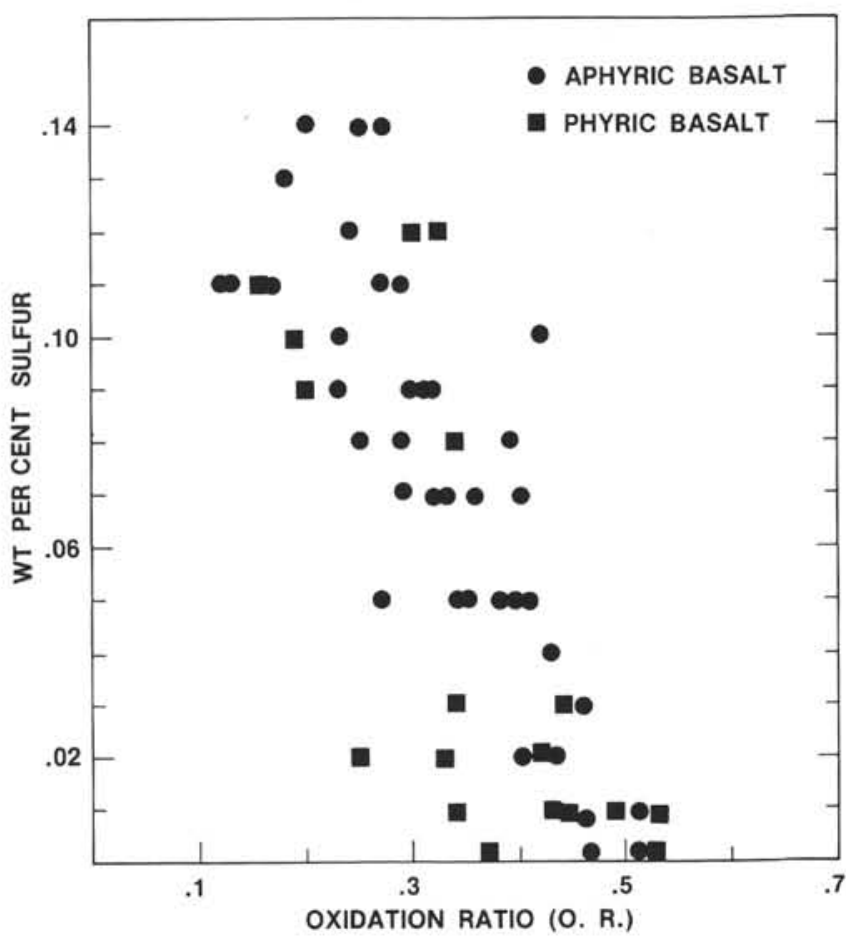

Figure 3. Relationship of sulfur content to oxidation ratio (O.R.) in Leg 45 basalts. The juvenile sulfur content of the basalts is inferred to have been greater than 0.14 per cent. 
TABLE 3 - Continued

\begin{tabular}{|c|c|c|c|c|c|c|}
\hline $395 \mathrm{~A}-14-2,140-147$ & $395 \mathrm{~A}-15-4,10-15$ & $395 \mathrm{~A}-15-4,16-20$ & $395 \mathrm{~A}-15-5,16-20$ & $395 \mathrm{~A}-15-5,16-20$ & $395 \mathrm{~A}-19-1,132-136$ & $395 \mathrm{~A}-21-1,87-90$ \\
\hline 125 & $128(1)$ & $128(2)$ & 130 & 130 & 132 & 133 \\
\hline 186 & 199 & 199 & 201 & 201 & 231 & 244 \\
\hline $\mathrm{P}_{2}$ & $\mathrm{P}_{2}$ & $\mathrm{P}_{2}$ & $\mathrm{P}_{2}$ & $\mathrm{P}_{2}$ & $P_{3}$ & $\mathrm{P}_{3}$ \\
\hline 31.1 & 31.5 & 32.6 & 28.2 & 29.0 & 32.5 & 32.4 \\
\hline 220 & 290 & 250 & 260 & 300 & 377 & 380 \\
\hline 87 & 83 & 90 & 100 & 70 & 110 & 130 \\
\hline 2.93 & 2.79 & 2.95 & 2.42 & 2.40 & 2.02 & 2.07 \\
\hline 9.5 & 9.2 & 9.7 & 8.0 & 7.8 & 6.7 & 6.8 \\
\hline 3.20 & 3.05 & 3.26 & 2.66 & 2.68 & 2.38 & 2.45 \\
\hline 1.17 & 1.16 & 1.20 & 1.01 & 1.03 & 0.91 & 0.93 \\
\hline 0.82 & 0.71 & 0.76 & 0.64 & 0.69 & 0.66 & 0.62 \\
\hline 3.0 & 2.7 & 2.8 & 2.4 & 2.4 & 2.4 & 2.4 \\
\hline 0.45 & 0.42 & 0.46 & 0.37 & 0.36 & 0.36 & 0.37 \\
\hline \multirow[t]{2}{*}{2.4} & 2.4 & 2.6 & 2.1 & 1.9 & 1.9 & 1.9 \\
\hline & $395 \mathrm{~A}-56-3,62-65$ & $395 \mathrm{~A}-57-1,40-43$ & $395 \mathrm{~A}-61-1,142-150$ & $395 \mathrm{~A}-63-4,86-93$ & $395 \mathrm{~A}-66-1,36-41$ & $395 \mathrm{~A}-67-\mathrm{CC}, 130-135$ \\
\hline 153 & 154 & 155 & 159 & 162 & 166 & 168 \\
\hline 539 & 567 & 573 & 609 & 626 & 645 & 660 \\
\hline$A_{3}$ & $\mathrm{~A}_{3}$ & $\mathrm{~A}_{4}$ & $\mathrm{P}_{4}$ & $\mathrm{P}_{4}$ & A4 & $\mathrm{A}_{4}$ \\
\hline 37.8 & 38.3 & 36.7 & 35.6 & 30.6 & 36.7 & 36.6 \\
\hline 280 & 260 & 340 & 320 & 380 & 320 & 330 \\
\hline 120 & 120 & 170 & 110 & 140 & 140 & 140 \\
\hline 3.81 & 4.24 & 3.34 & 2.24 & 2.30 & 3.18 & 3.37 \\
\hline 12.1 & 12.6 & 10.4 & 8.2 & 8.4 & 10.3 & 10.6 \\
\hline 4.33 & 4.20 & 3.67 & 2.81 & 2.93 & 3.56 & 3.67 \\
\hline 1.36 & 1.51 & 1.34 & 1.08 & 1.08 & 1.39 & 1.33 \\
\hline 1.02 & 1.06 & 0.93 & 0.72 & 0.72 & 0.99 & 0.94 \\
\hline 3.9 & 3.9 & 3.3 & 2.5 & 2.6 & 3.2 & 3.6 \\
\hline 0.59 & 0.58 & 0.53 & 0.38 & 0.43 & 0.47 & 0.50 \\
\hline 3.2 & 3.2 & 3.1 & 2.2 & 2.4 & 2.8 & 3.3 \\
\hline
\end{tabular}

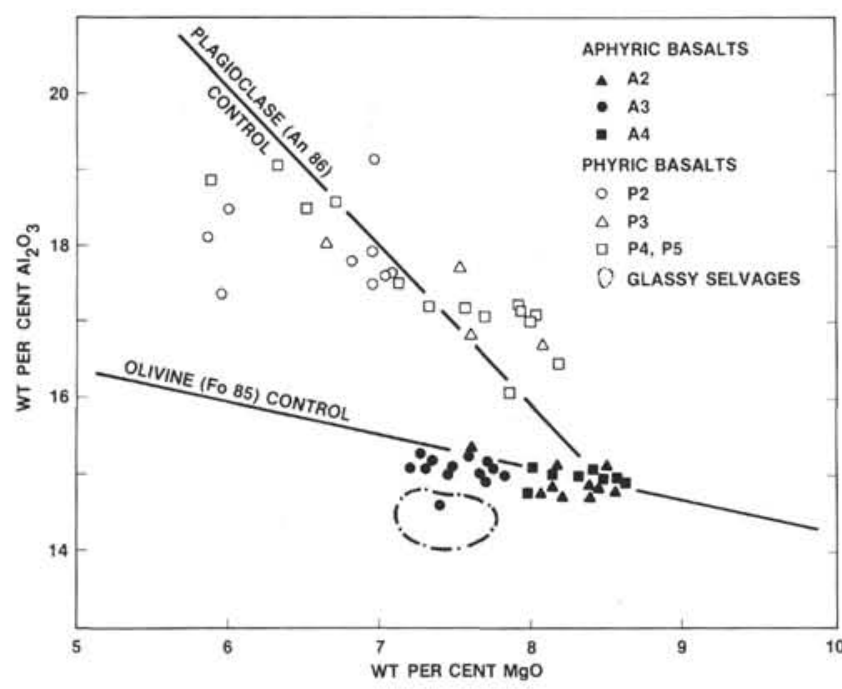

Figure 4. $\mathrm{Al}_{2} \mathrm{O}_{3}$ versus $\mathrm{MgO}$ for Leg 45 basalts. The olivine and plagioclase control lines are for illustrative purposes only; they are not intended as lines of "best fit," or to imply that the basalts are related simply by olivine and plagioclase fractionation.

basalts, both in absolute terms and with respect to other magmaphile elements. Consequently, $\mathrm{Sr} / \mathrm{Zr}$ ratios are distinctly different in the two basalt types, and vary widely in the phyric basalts ( 1.55 to 2.46 ), in contrast to the lower, essentially constant values (1.02 to 1.18 ) in the aphyric basalts.

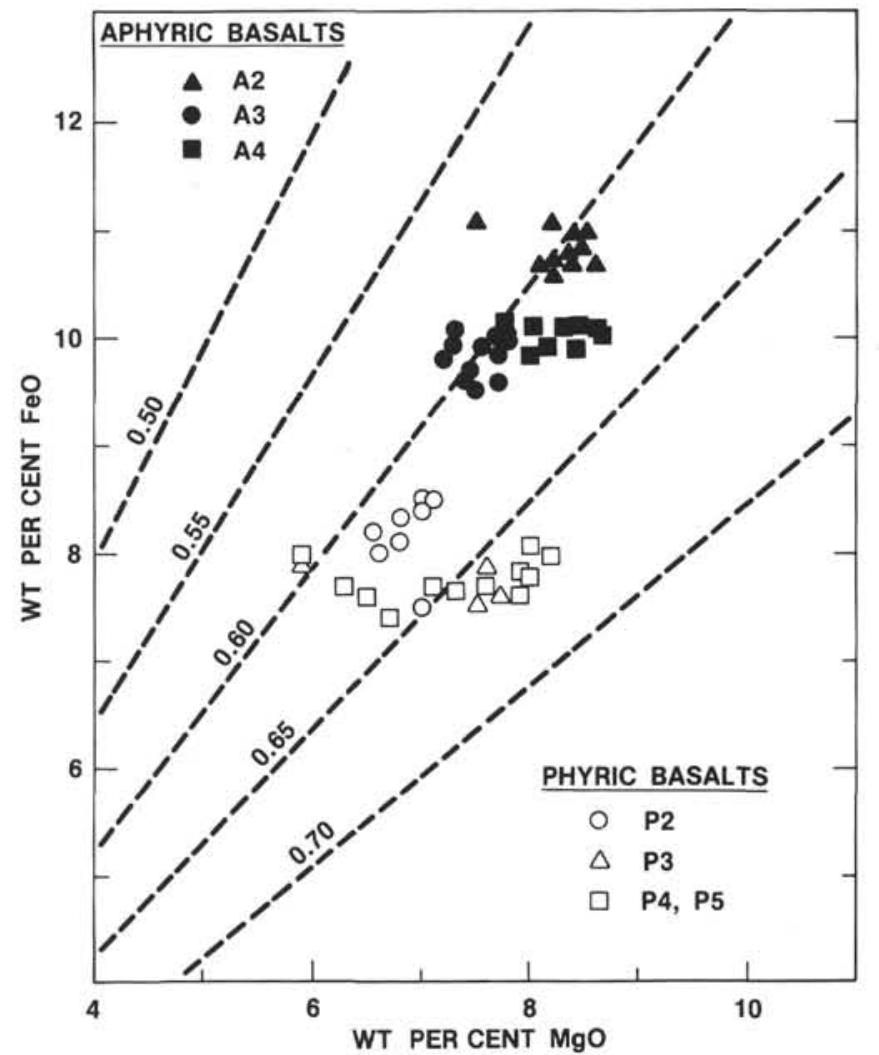

Figure 5. $\mathrm{MgO}-\mathrm{FeO}$ relationships for Leg 45 basalts. The dashed lines indicate $\mathrm{Mg}^{\prime}$-values from 0.5 to 0.7 . 
Figure 1 illustrates the normative olivine, plagioclase, and pyroxene relationships for these basalts, relative to the inferred olivine-plagioclase cotectic of Shido et al. (1971). All the aphyric basalts plot in the olivine tholeiite field, within a tightly controlled elongate group sub-parallel to the inferred cotectic. In contrast, the phyric basalts plot predominantly within the plagioclase tholeiite field and are widely scattered. Those phyric basalts plotting close to the inferred cotectic, or just within the olivine tholeiite field, have the lowest $\mathrm{Al}_{2} \mathrm{O}_{3}$ contents. Presumably, they contain fewer plagioclase phenocrysts than the majority of samples, and may be closer to melt compositions. As might be expected from these relationships, in a oneatmosphere melting experiment on a typical phyric basalt (\#138), plagioclase is the first phase to crystallize, followed by olivine, whereas in a typical aphyric basalt (\#122), olivine is the liquidus phase, followed by plagioclase (Dungan et al., this volume).

Initial studies, based on shipboard X-ray fluorescence analyses, recognized within the two major basalt types 10 compositionally distinct magmatic units. These are, in order of increasing depth below the sediment/ basement interface, A1, A2, P1, P2, P3, P4, P5, A3, $\mathrm{A} 4, \mathrm{P} 4, \mathrm{~A} 4$, and A5, where A denotes aphyric basalt and $\mathrm{P}$ phyric basalt.

Our data essentially confirm these subdivisions, subject to minor revisions in light of the more recent and comprehensive data. Average values, calculated from the data in Table 1, are given in Table 4. In order of increasing depth within Hole 395A, the compositional units following can be identified.

TABLE 4

Average Compositions of Leg 45 Basalt Types

\begin{tabular}{|c|c|c|c|c|c|c|c|c|}
\hline \multicolumn{4}{|c|}{ Aphyric Basalts } & \multicolumn{5}{|c|}{ Phyric Basaits } \\
\hline & A2 & A3 & A4 & P2 & P3 & P4 & P4 Sill & PS \\
\hline \multicolumn{9}{|c|}{ Major Elements (wt \%) } \\
\hline $\mathrm{SiO}_{2}$ & 48.96 & 49.28 & 48.07 & 49.16 & 49.27 & 49.22 & 49.15 & 49.13 \\
\hline $\mathrm{TiO}_{2}$ & 1.61 & 1.71 & 1.58 & 1.31 & 1.07 & 1.16 & 1.11 & 1.14 \\
\hline $\mathrm{Al}_{2} \mathrm{O}_{3}$ & 14.90 & 15.07 & 14.98 & 17.98 & 17.54 & 17.77 & 16.81 & 18.08 \\
\hline $\mathrm{FeO}^{*}$ & 10.81 & 9.84 & 10.01 & 8.11 & 7.85 & 7.71 & 7.90 & 7.74 \\
\hline $\mathrm{MnO}$ & 0.19 & 0.18 & 0.18 & 0.14 & 0.14 & 0.15 & 0.16 & 0.14 \\
\hline $\mathrm{MgO}$ & 8.27 & 7.48 & 8.36 & 6.75 & 7.27 & 7.20 & 7.76 & 7.03 \\
\hline $\mathrm{CaO}$ & 10.28 & 11.03 & 10.71 & 11.68 & 12.50 & 12.22 & 11.83 & 12.07 \\
\hline $\mathrm{Na}_{2} \mathrm{O}$ & 2.89 & 2.85 & 2.82 & 2.78 & 2.40 & 2.70 & 2.65 & 2.75 \\
\hline $\mathrm{K}_{2} \mathrm{O}$ & 0.15 & 0.23 & 0.16 & 0.10 & 0.13 & 0.15 & 0.11 & 0.16 \\
\hline $\mathrm{P}_{2} \mathrm{O}_{5}$ & 0.13 & 0.16 & 0.13 & 0.11 & 0.09 & 0.11 & 0.09 & 0.10 \\
\hline $\mathrm{s}^{203}$ & 0.11 & 0.06 & 0.04 & 0.09 & 0.02 & 0.01 & 0.04 & 0.02 \\
\hline Mg'-value & 0.602 & 0.601 & 0.623 & 0.622 & 0.647 & 0.649 & 0.661 & 0.643 \\
\hline \multicolumn{9}{|c|}{ Trace Elements (ppm) } \\
\hline $\mathbf{R b}$ & 1.4 & 2.3 & 1.6 & 0.9 & 1.9 & 2.0 & 2.3 & 2.5 \\
\hline $\mathrm{St}$ & 116 & 130 & 125 & 154 & 114 & 139 & 129 & 154 \\
\hline $\mathrm{Y}$ & 34.9 & 36.5 & 34.4 & 27.7 & 23.0 & 24.8 & 23.8 & 24.5 \\
\hline $\mathrm{Zr}$ & 105 & 119 & 108 & 88 & 63 & 74 & $70^{\circ}$ & 75 \\
\hline $\mathrm{Nb}$ & 1.9 & 2.2 & 2.1 & 1.5 & 1.2 & 1.2 & 0.7 & 1.1 \\
\hline La & 3.05 & 3.94 & 3.30 & 2.70 & 2.05 & 2.37 & 2.27 & 2.48 \\
\hline $\mathrm{Ce}$ & 10.1 & 12.2 & 10.4 & 8.9 & 6.7 & 7.7 & 8.3 & 8.1 \\
\hline $\mathrm{Sm}$ & 3.55 & 4.20 & 3.63 & 2.99 & 2.42 & 2.75 & 2.87 & 2.82 \\
\hline $\mathrm{Eu}$ & 1.33 & $\begin{array}{l}1.44 \\
1.44\end{array}$ & 1.35 & 1.11 & 0.92 & 1.05 & 1.08 & 1.06 \\
\hline $\mathrm{Tb}$ & 0.94 & $\begin{array}{l}1.04 \\
\text {. }\end{array}$ & 0.95 & 0.73 & 0.64 & 0.76 & 0.72 & 0.67 \\
\hline $\mathrm{Yb}$ & 3.5 & 3.8 & 3.4 & 2.7 & 2.4 & 2.6 & 2.5 & 2.7 \\
\hline $\mathrm{Lu}$ & 0.51 & 0.57 & 0.50 & 0.41 & 0.36 & 0.40 & 0.40 & 0.41 \\
\hline $\mathrm{Hf}$ & 2.9 & 3.2 & 3.1 & $\begin{array}{l}.41 \\
2.3\end{array}$ & $\begin{array}{l}1.90 \\
1.9\end{array}$ & 2.3 & 2.3 & 2.2 \\
\hline $\mathrm{Sc}$ & 36.9 & 38.4 & 36.7 & 30.4 & 32.5 & 33.7 & 33.1 & 32.3 \\
\hline $\mathrm{Cr}$ & 295 & 280 & 330 & 262 & 379 & 340 & 350 & 310 \\
\hline $\mathrm{Ni}$ & 150 & 98 & 150 & 90 & 120 & 140 & 125 & 140 \\
\hline $\mathrm{La} / \mathrm{Sm}$ & 0.86 & 0.94 & 0.91 & 0.90 & 0.85 & 0.86 & 0.79 & 0.88 \\
\hline $\mathrm{La} / \mathrm{Yb}$ & 0.87 & 1.04 & 0.97 & 1.00 & 0.85 & 0.91 & 0.91 & 0.92 \\
\hline $\mathrm{Sm} / \mathrm{Eu}$ & 2.67 & 2.92 & 2.69 & 2.69 & 2.63 & 2.62 & 2.66 & 2.66 \\
\hline $\mathrm{Sr}_{\mathrm{Z}} / \mathrm{Zr}_{\mathrm{r}}$ & 1.10 & 1.09 & 1.16 & 1.75 & 1.81 & 1.88 & 1.84 & 2.05 \\
\hline $\mathrm{Zt} / \mathrm{Nb}$ & 55 & 54 & 51 & 59 & 53 & 62 & 100 & 68 \\
\hline $\mathrm{Z} / \mathrm{Y}$ & 3.01 & 3.26 & 3.14 & 3.2 & 2.7 & 3.0 & 2.9 & 3.1 \\
\hline $\mathrm{Ti} / \mathrm{Zr}_{\mathrm{r}}$ & 92 & 86 & 88 & 89 & 102 & 94 & 95 & 91 \\
\hline $\mathrm{Zt} / \mathrm{Hf}$ & 36 & 37 & 35 & 38 & 33 & 32 & 30 & 34 \\
\hline
\end{tabular}

Aphyric Unit A2 (106 to $173 \mathrm{~m}$ ) - This is the uppermost aphyric unit, and occurs in the upper 29 meters of Hole 395, as well as in Hole 395A. It consists of a thick sequence of chemically homogeneous, rapidly chilled pillow basalts, and has a characteristic variolitic texture containing microphenocrysts of olivine. This basalt type is distinguished from the other aphyric basalts principally on the basis of higher iron concentrations ( 10.6 to $11.1 \%$ ) and by slightly lower abundances of strontium (Table 4). The compositional variation is small, and is commensurate with minor $(<5 \%)$ olivine fractionation.

In the initial shipboard studies, the magmatic Unit Al was based on a single sample (11-1, 103-108 cm) from near the top of basement in Hole 395. It was more iron-rich than underlying members of the A2 basalts. In order to locate the A1/A2 boundary we have analyzed basalts immediately above and below this sample, and find that they have typical A2 compositions (Table 1, \#109, 110). From this we conclude that Unit A1 is not a distinct magmatic unit, and that the iron-rich sample analyzed during shipboard studies is a more evolved variant of the A2 basalt type.

Phyric Unit P2 (173 to $201 \mathrm{~m}$ )-This unit occurs at the top of the thick 181-meter sequence of phyric basalts that were cored in Hole 395A, and also occurs in the bottom 10 meters of Hole 395. In Hole 395A, almost all of this unit is composed of a single thick ( 25 $\mathrm{m})$, massive cooling unit. Except for the lowermost sample in the cooling unit (Table 1, \#130), the P2 basalts are uniform in composition, and are more evolved than the other phyric basalts. This is reflected in lower $\mathrm{Mg}^{\prime}$ values and higher abundances to $\mathrm{TiO}_{2}$ and other magmaphile elements (Figure 2). The lowermost sample in the cooling unit is higher in $\mathrm{Al}_{2} \mathrm{O}_{3}$, has a higher $\mathrm{Mg}^{\prime}$ value, and has correspondingly lower abundances of magmaphile elements. In many respects it resembles other, more "primitive" phyric basalts lower in the stratigraphic sequence. We attribute this difference to either preferential accumulation of plagioclase and olivine phenocrysts at the base of the cooling unit, or upward migration of interstitial magma, caused by filter pressing.

Phyric Unit P3 (201 to $260 \mathrm{~m}$ )-This unit is distinguished from the other phyric units by lower $\mathrm{TiO}_{2}$ and magmaphile element abundances (Figures 2 and 6). Strontium is also low in this unit (110 to $119 \mathrm{ppm}$ ), relative to all other phyric basalts. The plagioclaseolivine phyric basalt associated with the brecciated serpentinized periodotite zone in Hole 395 is also low in strontium (Table 1, \#115), and tends to be lower in magmaphile elements than most other phyric basalts. In many respects, it closely resembles the P3 basalt type in Hole 395A, and may simply be fragments of this basalt type incorporated into the breccia zone, rather than a separate magmatic unit (P1) as was suggested by the shipboard studies.

Phyric Units P4 and P5 (260 to $356 \mathrm{~m}$ )-Initial shipboard analyses indicated that these two basalt types are essentially similar in bulk chemistry, but 


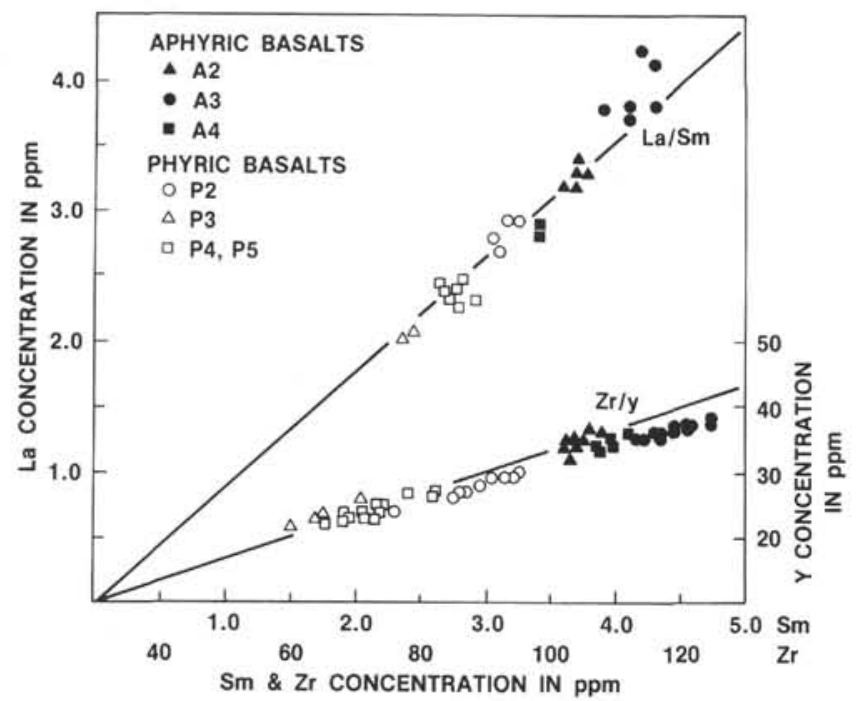

Figure 6. Lanthanum-Samarium and ZirconiumYttrium relationships in Leg 45 basalts. The lines shown are for constant $\mathrm{La} / \mathrm{Sm}$ and $\mathrm{Zr} / \mathrm{Y}$ ratios.

differ in strontium content. Additional analyses reported here (Table 1) show that both units contain strontium in a wide range of values: strontium in P4 varies between 122 and $165 \mathrm{ppm}$, and in P5 between 132 and $175 \mathrm{ppm}$. Consequently, we believe that the initial distinction between these two basalt types is no longer tenable.

As a group these basalts are highly variable both in $\mathrm{Al}_{2} \mathrm{O}_{3}$ content, which varies from 16.1 to 19.1 per cent, and in $\mathrm{Mg}^{\prime}$-values (between 0.59 and 0.67 per cent). Much of this variability must be attributed to localized variation in plagioclase and olivine phenocryst content.

Deeper in the section (608 to $629 \mathrm{~m}$ ), there is a massive $(21 \mathrm{~m})$ cooling unit chemically equivalent to the P4-P5 phyric basalts. We believe this to be a sill that intruded underlying aphyric basalts penecontemporaneously with extrusion of the P4-P5 basalts.

Aphyric Unit A3 (356 to $570 \mathrm{~m}$ )- This exceptionally thick sequence of compositionally uniform pillow basalts is more evolved than the other aphyric units. These basalts are multiply saturated with microphenocrysts of both olivine and plagioclase (Dungan et al., this volume), and plot towards the low-temperature end of the inferred cotectic in Figure 1. Although the $\mathrm{Mg}^{\prime}$-values compare closely with those of the A2 basalts (e.g., 0.59 to 0.61 ), they are distinctly lower in both $\mathrm{FeO}$ and $\mathrm{MgO}$ (Figure 5), and on the basis of fig. 7 in Roeder and Emslie (1970), can be inferred to have lower liquidus temperatures. In keeping with their evolved nature, these basalts have the highest lithophile element abundances, and are characterized by a more distinct $\mathrm{Eu}$ anomaly than is observed for the other aphyric basalts. The unit as a whole exhibits little compositional variation, and there are no obvious fractionation trends.

At the sediment/basement interface in Hole 395A is a rubble zone containing fragments of aphyric basalt, serpentinized peridotite, and gabbro (Cores 4 and 5).
Two of these basalts are indistinguishable from the A3 basalts (Table $1, \# 117,118$ ); this suggests that this basalt type is exposed at the surface of the basement within close proximity to the drill site.

Aphyric Unit A4 (570 to $608 \mathrm{~m}$ and 629 to $657 \mathrm{~m}$ )-This unit is intruded by a 21-meter sill compositionally equivalent to the P4-P5 phyric basalts. There is no appreciable difference in chemistry between the basalts above the sill (Table 1, \#155-160) and those beneath (Table 1, \#161-168). All are aphyric basalts containing olivine microphenocrysts. They are both texturally and chemically similar to the A2 basalts, but can be distinguished from them by a tendency toward higher normative olivine contents (Figure 1), higher $\mathrm{Mg}^{\prime}$ values $(0.60$ to 0.63$)$, and lower total iron concentrations for a given value of $\mathrm{MgO}$ (Figure 5). $\mathrm{TiO}_{2}, \mathrm{Sr}$, and the magmaphile element abundances are essentially identical to those of the $\mathrm{A} 2$ basalts.

\section{DISCUSSION}

The chondrite-normalized rare-earth abundances for the Leg 45 samples (Figure 7) show them to be "normal" LIL-element-depleted basalts. They are light-rare-earth depleted, and have $\mathrm{La} / \mathrm{Sm}$ and $\mathrm{La} / \mathrm{Yb}$ ratios less than 1.0 (Table 4 ), similar to the Type I basalts of Bryan et al., (1976). The Nb content of these basalts is low, resulting in high $\mathrm{Zr} / \mathrm{Nb}$ ratios (mean = 58) similar to those in other LIL-element-depleted basalts (Pearce and Cann, 1973; Erlank and Kable 1976; Rhodes et al., 1976), and considerably higher than the $\mathrm{Zr} / \mathrm{Nb}$ ratios $(<10)$ of oceanic islands and "anomalous" Type II basalts sampled on the MidAtlantic Ridge at $45^{\circ} \mathrm{N}$ and in the FAMOUS area (Erlank and Kable, 1976; Rhodes et al., unpublished data). Two ${ }^{87} \mathrm{Sr} /{ }^{86} \mathrm{Sr}$ values of $0.70276 \pm 10$ and $0.70280 \pm 8$, obtained for Samples 117 and 153, respectively, are also characteristic of "normal," Type I LILelement-depleted basalts (Hart, 1976).

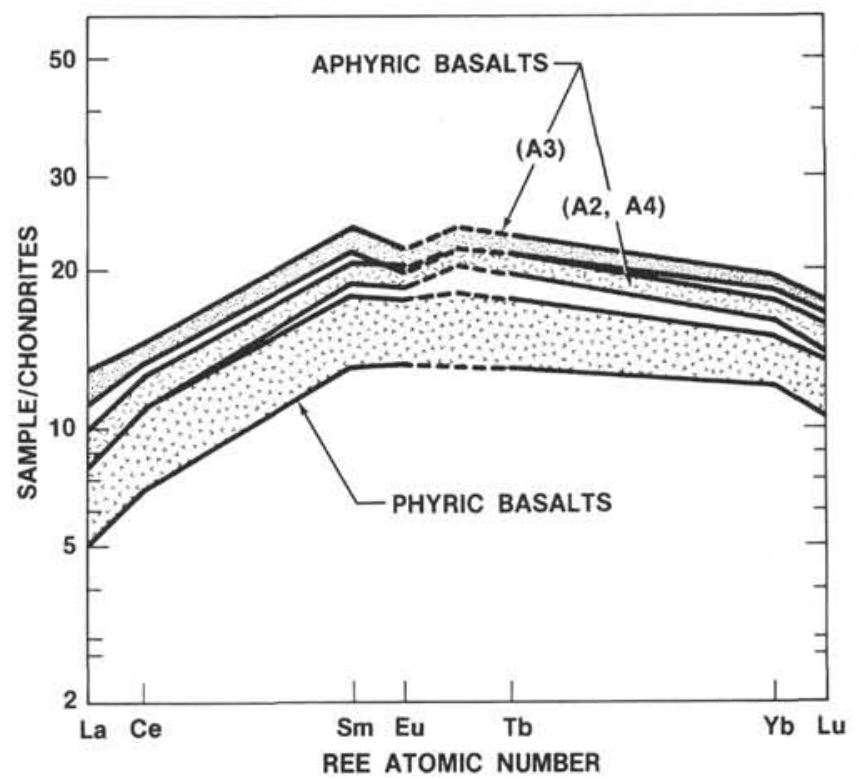

Figure 7. Chrondite-normalized abundances in Leg 45 basalts. 
Inspection of Figures 6 and 7 and Table 4 shows that most magmaphile element ratios are very similar for the various basalt types, and do not differ substantially between the phyric and aphyric basalts. An obvious exception is the ratio of strontium to other magmaphile elements. This is illustrated by the $\mathrm{Sr} / \mathrm{Zr}$ ratio, which is constant in the aphyric basalts, where $\mathrm{Sr}$ behaves as a magmaphile element, but is both distinctly different and highly variable in the phyric basalts, where plagioclase influence is prevalent. Since many magmaphile element ratios and the characteristic REE patterns are not substantially changed by fractional crystallization involving the observed phenocryst phases, olivine, and plagioclase (Kay et al., 1970; Schilling 1971; Frey et al., 1974; Schilling, 1975), we deduce that these ratios reflect the characteristics of the mantle source region, and that all of the various basalt types were derived from an essentially similar and homogeneous LIL-element-depleted mantle source. Similar ratios pertain to basalts from Holes 396 (Table 2) and 396B (Dungan et al., Initial Reports of the Deep Sea Drilling Project, v. 46, in preparation); this implies a widespread and homogeneous mantle source for basaltic vulcanism along this section of the MidAtlantic Ridge. This contrasts markedly with the wide variations in magmaphile element ratios found in Leg 37 basalts, both within and between holes (Blanchard et al., 1976), and the consequent necessity to postulate a complex and heterogeneous mantle source for the basalts in that region.

Although these basalts appear ultimately to have been derived by partial melting of a common source, all are evolved and have undergone moderate amounts of differentiation. Evidence of this is provided by the low $\mathrm{Mg}^{\prime}$-values ( 0.57 to 0.67$)$, low Ni concentrations ( $<190 \mathrm{ppm})$, moderate magmaphile element abundances (e.g., $\mathrm{TiO}_{2}=1.0$ to $1.7 \%$ ), and the presence of multiple phenocryst phases in all but the A2 and A4 basalts. Since the compositional variation within the individual basalt groups is small, and there are no clearly defined internal fractionation trends, we suggest that magma compositions were established by differentiation within shallow magma chambers, followed by episodic eruption onto the sea floor, with little or no fractionation at the surface or enroute to the surface.

There can be little doubt that the compositions of the glassy and variolitic aphyric basalts are close to those of magmatic liquids. The compositions of the phyric basalts are more enigmatic. Several lines of evidence indicate that these rocks are far removed from liquid compositions: (a) Compilations of basaltic glass compositions (Melson et al., 1976) indicate that magmatic liquids with more than 17 per cent $\mathrm{Al}_{2} \mathrm{O}_{3}$ are extremely rare. Similarly, the glassy selvages on the phyric basalts (unpublished data) are much lower in $\mathrm{Al}_{2} \mathrm{O}_{3}$ and not substantially different from the aphyric basalts (Figure 4). (b) The wide scatter of compositions in the normative olivine-plagioclase-pyroxene diagram (Figure 1) suggests plagioclase and olivine addition to liquids close to the inferred olivine-plagio- clase cotectic. Plagioclase addition trends are also evident in several other variation diagrams (e.g., Figures 4 and 5). (c) Dungan et al. (this volume) present experimental, textural, and compositional evidence indicating that many of the plagioclase and olivine phenocrysts are too anorthitic or forsteritic to have crystallized from melts having the compositions of the phyric basalts, but derive instead from more "primitive" basalts.

From this evidence, we think that the phyric basalts have a mixed heritage, resulting from mixing of consanguineous "primitive" and evolved magmas and their attendant phenocrysts. If this interpretation is correct, the higher $\mathrm{Mg}^{\prime}$-values, normative $\mathrm{An} /$ $(A b+A n)$ ratios, and lower magmaphile element abundances of the phyric basalts relative to the aphyric ones result in part from the addition of up to 20 per cent plagioclase and 5 per cent olivine phenocrysts. The glassy rims on phyric basalt pillows probably provide the most reliable estimate of the melt compositions. They have $\mathrm{Mg}^{\prime}$-values comparable to those of the more evolved aphyric basalts $(<0.60)$, but are distinguished from them by lower $\mathrm{TiO}_{2}$ values (Figure 2) and by higher $\mathrm{CaO} / \mathrm{Al}_{2} \mathrm{O}_{3}$ ratios. Thus, the phyric basalts are not simply related to any of the aphyric basalts types by processes of plagioclase and olivine accumulation.

An understanding of the relationships between the various phyric basalt units is also frustrated by uncertainty concerning melt compositions. In terms of increasing magmaphile element abundances, the basalt types are ranked as follows: P3, P4-5, P2. This is not simply a function of decreasing plagioclase and olivine accumulation, but reflects a fundamental property of each of the basalt types. The increase in magmaphile element abundances from P3 to P2 is too large and varied, however, to be accounted for simply by crystal fractionation of the dominant phenocryst phases, plagioclase and olivine, and cannot readily be reconciled with the absence of systematic changes in major element chemistry. We attribute these differences in the magmaphile element abundances in the phyric basalts to discrete magma types, containing varying proportions of admixed plagioclase, olivine, and rarer clinopyroxene phenocrysts.

The relationships between the three aphyric basalt units are less complicated. The A2 and A4 basalts are broadly similar in major- and trace-element chemistry, and both contain only olivine as the microphenocryst phase. The A2 basalts are higher in total iron and tend to have lower $\mathrm{Mg}^{\prime}$-values than the majority of the A4 basalts (Figure 5). Consequently, these two basalt types cannot be related by olivine fractionation, the only observed phenocryst phase, and the liquidus phase in equilibrium melting experiments (Dungan et al., this volume). The A3 basalts contain microphenocrysts of both olivine and plagioclase, and are more evolved than the stratigraphically lower A4 basalts, from which they may have been derived by olivine fractionation. The magmaphile element abundances, 
however, are at variance with this interpretation. Some elements, such as $\mathrm{Sr}, \mathrm{Y}$, and $\mathrm{Sc}$ are increased in the A3 basalts relative to the $\mathrm{A} 4$ basalts, commensurate with the 5 per cent olivine and plagioclase fractionation required by the major-element data. The differences in other elements (e.g., Ti, Zr, La, Sm, Yb) are, however, too large to have resulted from such a small amount of fractionation. Further, the amount of fractionation estimated from the reciprocal of the ratio of magmaphile elements in the A3 to the A4 basalts (Anderson and Greenland, 1969) is not constant for the different elements, and varies from as little as 4 per cent for $\mathrm{Sr}$ to as much as 20 per cent for Sm. Clearly, these magma types are not related directly by crystal fractionation processes.

\section{CONCLUSION}

We have, at the Leg 45 site, several discrete magma types, both phyric and aphyric, not related directly by crystal fractionation, which appear to have been derived from a common mantle source. The prevailing interpretation of such relationships is that each basalt type represents an evolved derivative of more "primitive" basaltic magmas, resulting from varying degrees of melting of a common mantle source. The evidence presented here, of magma mixing in the phyric basalts, suggests that such an interpretation may be too simple. We suggest instead that magma mixing of consanguineous evolved and "primitive" basalts, together with their attendant phenocrysts, may better explain many characteristics of the basalts. A subsequent publication documenting the role of magma mixing in these and

other ocean floor basalts is in preparation (Rhodes et al., in press).

\section{REFERENCES}

Anderson, A. T. and Greenland, L. P., 1969. Phosphorus fractionation diagram as a quantitative indicator of crystallization differentiation of basaltic liquids, Geochim. Cosmochim. Acta, v. 33, p. 493-505.

Bass, M. N., 1971. Variable abyssal basalt populations and their relation to sea-floor spreading rates, Earth Planet. Sci. Lett., v. 11, p. 18-22.

Blanchard, D. P., Rhodes, J. M., Dungan, M. A., Rodgers, K. V., Donaldson, C. H., Brannon, J. C., Jacobs, J. W., and Gibson, E. K., 1976. The chemistry and petrology of basalts from Leg 37 of the Deep Sea Drilling Project, $J$. Geophys. Res., v. 81, p. 4231-4246.

Bryan, W. B. and Moore, J. G., 1976. Compositional variations of young basalts in the Mid-Atlantic Ridge rift valley near lat. $46^{\circ} 49^{\prime}$ N, Geol. Soc. Am. Bull., v. 88, p. 556-570.

Bryan, W. B., Thompson, G., Frey, F. A., and Dickey, J. J., 1976. Inferred settings and differentiation in basalts from the Deep Sea Drilling Project, J. Geophys. Res., v. 81, p. 4285-4304.

Engel, A. E., Engel, C. G., and Havens, R. G., 1965. Chemical characteristics of oceanic basalts and the upper mantle, Geol. Soc. Am. Bull., v. 76, p. 719-734.

Erlank, A. J. and Kable, E. J. D., 1976. The significance of incompatible elements in Mid-Atlantic Ridge basalts from $45^{\circ} \mathrm{N}$, with particular reference to $\mathrm{Zr} / \mathrm{Nb}$, Contrib. Mineral. Petrol., v. 54, p. 281-291.
Frey, F. A., Bryan, W. B., and Thompson, G., 1974. Atlantic ocean floor: Geochemistry and petrology of basalts from Legs 2 and 3 of the Deep Sea Drilling Project, J. Geophys. Res., v. 79, p. 5507-5527.

Hart, S. R., 1976. LIL-element geochemistry, Leg 34 basalts. In Hart, S. R., Yeats, R. S., et al., Initial Reports of the Deep Sea Drilling Project, v. 34: Washington (U.S. Government Printing Office), p. 283-288.

Jacobs, J. W., Korotev, R. L., Blanchard, D. P., and Haskin, L. A., in press. A well tested procedure for instrumental neutron activation analysis of silicate rocks and minerals, J. Radioanal. Chem.

Kay, R., Hubbard, N. J., and Gast, P. W., 1970. Chemical characteristics and origin of oceanic ridge volcanic rocks, J. Geophys. Res., v. 75, p. 1585-1613.

Maxwell, J. A., 1968. Rock and mineral analysis: New York (Interscience), p. 419-421.

Melson, W. G., Vallier, T. L., Wright, T. L., Byerly, G., and Nelen, J., 1976. Chemical diversity of abyssal volcanic glass erupted along Pacific, Atlantic, and Indian ocean sea-floor spreading centers. In The geophysics of the pacific ocean basin and its margin: Washington (American Geophysical Union), p. 351-367.

Moore, J. G. and Fabbi, B. P., 1971. An estimate of the juvenile sulfur content of basalt, Contrib. Mineral. Petrol., v. 33 , p. $118-127$.

Norrish, K. and Chappell, B. W. 1967. X-ray fluorescence spectrography. In Zussman, J. (Ed.), Physical methods in determinative mineralogy: New York (Academic Press), p. 161-214.

Norrish, K. and Hutton, J. T., 1969. An accurate X-ray spectrographic method for the analysis of a wide range of geological samples, Geochim. Cosmochim. Acta, v. 33, p. 431-453.

Pearce, J. A., and Cann, J. R., 1973. Tectonic setting of basic volcanic rocks determined using trace element analysis, Earth Planet. Sci. Lett., v. 19. p. 290-300.

Reynolds, R. C., 1967. Estimation of mass absorption coefficients by Compton scattering: Improvements and extensions of the method, Am. Mineralogist, v. 48, p. 11331143.

Rhodes, J. M., Blanchard, D. P., Rodgers, K. V., Jacobs, J. W., and Brannon, J. C., 1976. Petrology and chemistry of basalts from the Nazca Plate: Part 2-major and trace element chemistry. In Hart, S. R., Yeats, R. S., et al., Initial Reports of the Deep Sea Drilling Project, v. 34: Washington, D. C. (U.S. Government Printing Office), p. 239-244.

Rhodes, J. M., Dungan, M. A., Blanchard, D. P. and Long, P. E., in press. Magma mixing at mid-ocean ridges: Evidence from basalts drilled near $22^{\circ} \mathrm{N}$ on the Mid-Atlantic Ridge, Tectonophysics.

Roeder, P. L. and Emslie, P. F., 1970. Olivine-liquid equilibrium, Contrib. Mineral. Petrol., v. 29, p. 275-289.

Schilling, J. G., 1971. Sea-floor evolution: Rare-earth evidence, Phil. Trans. Roy. Soc. London, ser. A., v. 268, p. 663-706.

1975. Rare earth variations across 'normal segments' of the Reykjanes Ridge, $60^{\circ}-53^{\circ} \mathrm{N}$, Mid-Atlantic Ridge, $29^{\circ} \mathrm{S}$, and East Pacific rise $2^{\circ}-19^{\circ} \mathrm{S}$, and evidence on the composition of the underlying low velocity layer, J. Geophys. Res., v. 80, p. 1459-1473.

Shido, F. A., Miyashiro, A., and Ewing, M., 1971. Crystallization of abyssal tholeiites, Contrib. Mineral. Petrol., v. 31 , p. $251-266$

Yoder, H. S. and Tilley, C. E., 1962. Origin of basalt magmas: an experimental study of natural and synthetic rock systems, J. Petrol., v. 3, p. 342-532. 